\section{(A) Check for updates}

Cite this: Analyst, 2021, 146, 6416

\title{
Ophthalmic sensing technologies for ocular disease diagnostics
}

\author{
Yuqi Shi, (D) a Nan Jiang, (D)*b Priyanka Bikkannavar, (D) ${ }^{\mathrm{c}}$ M. Francesca Cordeiro (D) ${ }^{\mathrm{c}, \mathrm{d}}$ \\ and Ali K. Yetisen (D)*a
}

Point-of-care diagnosis and personalized treatments are critical in ocular physiology and disease. Continuous sampling of tear fluid for ocular diagnosis is a need for further exploration. Several techniques have been developed for possible ophthalmological applications, from traditional spectroscopies to wearable sensors. Contact lenses are commonly used devices for vision correction, as well as for other therapeutic and cosmetic purposes. They are increasingly being developed into ocular sensors, being used to sense and monitor biochemical analytes in tear fluid, ocular surface temperature, intraocular pressure, and $\mathrm{pH}$ value. These sensors have had success in detecting ocular conditions, optimizing pharmaceutical treatments, and tracking treatment efficacy in point-of-care settings. However, there is a paucity of new and effective instrumentation reported in ophthalmology. Hence, this review will summarize the applied ophthalmic technologies for ocular diagnostics and tear monitoring, including both conventional and biosensing technologies. Besides applications of smart readout devices for continuous monitoring, targeted biomarkers are also discussed for the convenience of diagnosis of various ocular diseases. A further discussion is also provided for future aspects and market requirements related to the commercialization of novel types of contact lens sensors.

Received 11th July 2021 Accepted 21st September 2021 DOI: $10.1039 / \mathrm{d} 1 \mathrm{an} 01244 \mathrm{~d}$ rsc.li/analyst of people with vision impairment are from developing countries and aged 50 years or older. ${ }^{4}$ The most common causes of visual impairment are refractive errors, cataracts, glaucoma, age-related macular degeneration, corneal opacification, diabetic retinopathy, childhood blindness and trachoma. In some cases, the cause of visual impairment remains undetermined. All of these causes can lead to blindness, and possibly be accompanied by other conditions including intellectual disability, cerebral palsy, hearing impairments, and epilepsy. However, approximately $80 \%$ of these visual impairments can be avoided if proper testing and diagnostic processes are carried out. ${ }^{3,4}$ Furthermore, the expenditure on ocular care and treatments is also enormous. The total cost of vision impairment globally had reached approximately $\$ 3$ trillion in 2011, comprising both direct and indirect health care costs, with $\$ 4030$ for each patient with ocular dysfunction. ${ }^{5}$ The largest direct medical cost was contributed by the hospitalization and utilization of medical services for diagnosis and treatment in the initial stages of vision impairment and blindness. ${ }^{6}$ Vision therapy and ocular diagnosis are well-researched areas globally, including in the United States, Australia, and European countries. Both clinical trials and fundamental labbased research are vital for ophthalmology. Furthermore, vision defects inevitably affect employment in the modern world. For example, vision testing and regulation are common

\footnotetext{
${ }^{a}$ Department of Chemical Engineering, Imperial College London, South Kensington, London, SW7 2BU, UK. E-mail: a.yetisen@imperial.ac.uk

${ }^{b}$ West China School of Basic Medical Sciences and Forensic Medicine, Sichuan University, Chengdu 610041, China. E-mail: jiangnansophia@scu.edu.cn ${ }^{c} U C L$ Institute of Ophthalmology, London, UK

${ }^{d}$ ICORG, Imperial College London, London, UK
} 


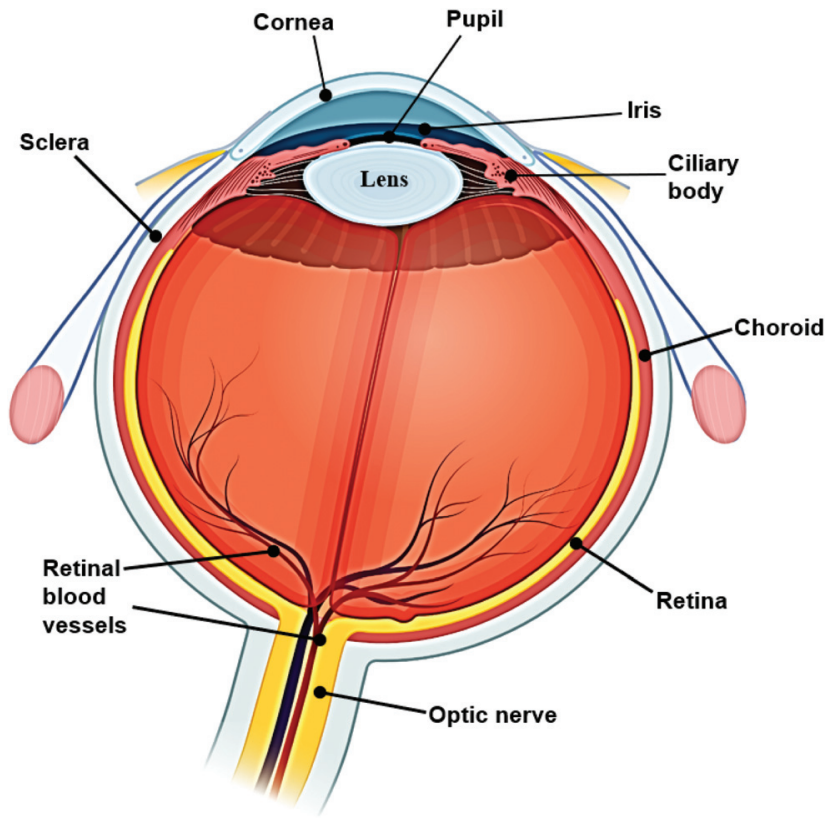

Fig. 1 Structure of human eyeball. The human eye comprises three layers. The outer layer, which involves the cornea and sclera, manages the entire shape of the eyeball and gives support to a deeper structure. The middle layer consists of the choroid, ciliary body, and iris. The retina is the inner layer and receives a blood supply from the central retinal artery and the posterior ciliary arteries.

for particular occupations, such as for defence force personnel. Hence, novel ocular diagnostic techniques have become an emerging market worldwide in recent years.

According to the global demographic of health report from the WHO, the sub-health population has reached $75 \%$. With the increasing demand for point-of-care (POC) diagnostics, it becomes crucial to develop rapid and accurate methods for detecting and monitoring biological and chemical molecules which may be present in minimal concentrations in physiological and pathological conditions. ${ }^{7}$ Biosensors are a typical example of this and can rapidly detect analyte levels. Many commercial sensors, such as wearable medical sensors have been developed to detect and continuously monitor blood pressure, heart rate, blood oxygen levels, and body temperature. The evolution of biosensor technologies provides this opportunity, and the obtained information is valuable for the monitoring and diagnosis of typical diseases, as well as for understanding pathological processes within these physiological systems. In the future, biosensors will be developed to test saliva, skin, interstitial fluid, and tear fluid for diagnostic purposes. $^{8-10}$ Tear fluid comprises similar constituents to body fluid such as serum or plasma, but is simpler in complexity. ${ }^{11}$ Since the last decade, studies have been conducted aiming to develop contact lens sensors and a POC platform for tear fluid monitoring and disease diagnosis. For instance, the possibility of diagnosing neurological diseases such as multiple sclerosis and Parkinson's disease via evaluation of tear fluid is being explored.
This review will summarize typical types of technologies that have been applied for ophthalmological diagnosis (Fig. 2), including the fundamental commercialized instruments, novel imaging technologies, and various laboratory-based biosensing techniques with accompanying experimental data, particularly focusing on different types of contact lens sensing technologies. Based on theoretical and applied clinical considerations, discussions aim to provide an overview of ocular disease diagnosis platforms, with a review of the overall market situation and future research directions.

\section{Diagnostic approaches}

\subsection{Spectroscopy technology}

Routine eye examination is an important step in the initial assessment of patients suspected to have ocular diseases, after which further investigations are carried out for specific diagnosis and monitoring. There are several quick common tests used by optometrists. Retinoscopy is a hand-held objective method that is widely used to measure the refractive error of the eye. Light shone into the eye is reflected from the retina. This reflected light is named the retinoscopy reflex. Two types of retinoscopes are utilized for clinical assessment: streak retinoscopes (Fig. 3A), and spot retinoscopes (Fig. 3B) which have similar operational principles (Fig. 3C). Also, trial lenses are used to provide refractive error results, ${ }^{12}$ which ultimately are optimized by manual refraction.

Among various imaging technologies, external eye photography is an obvious but not specialized method. The macrophotography is applied to record the external appearance of the eyes with the surrounding lids and facial structures. Therefore, it is commonly used to observe ocular surface lesions or surrounding issues and document pre- and post-surgical alignment of the eyes or eyelids. ${ }^{13}$ As part of a complete ocular examination, slit-lamp microscopy allows for better examination of structures of the eye, in particular the anterior segment of the eye, including the cornea, iris and lens (Fig. 1). A slit lamp microscope shines a very bright light and magnifies ocular structures, allowing detailed visualization and precise anatomical measurements. ${ }^{14}$ Eye drops are typically applied before the examination to dilate the pupils for closer examination of posterior structures. This examination can report the physical condition of ocular structures and aid the diagnosis of ocular diseases, such as cataract, glaucoma, uveitis, macular degeneration, and corneal or scleral injuries or damage. ${ }^{13-15}$ Images of the eye can also be accessed by various cameras (Fig. 4). ${ }^{16}$ Digital cameras have been successfully adapted to slit-lamp microscopy, providing high quality ocular images. Higher spatial resolutions have been found in cameras with a high-definition multimedia interface (HDMI), including cameras in smartphones. ${ }^{16}$ With the application of smartphone imaging, more possibilities can be explored for handheld read-out devices which will be helpful for POC uses. However, the highest resolution of ocular viewing is still achieved by using the slit-lamp bio-microscope. 


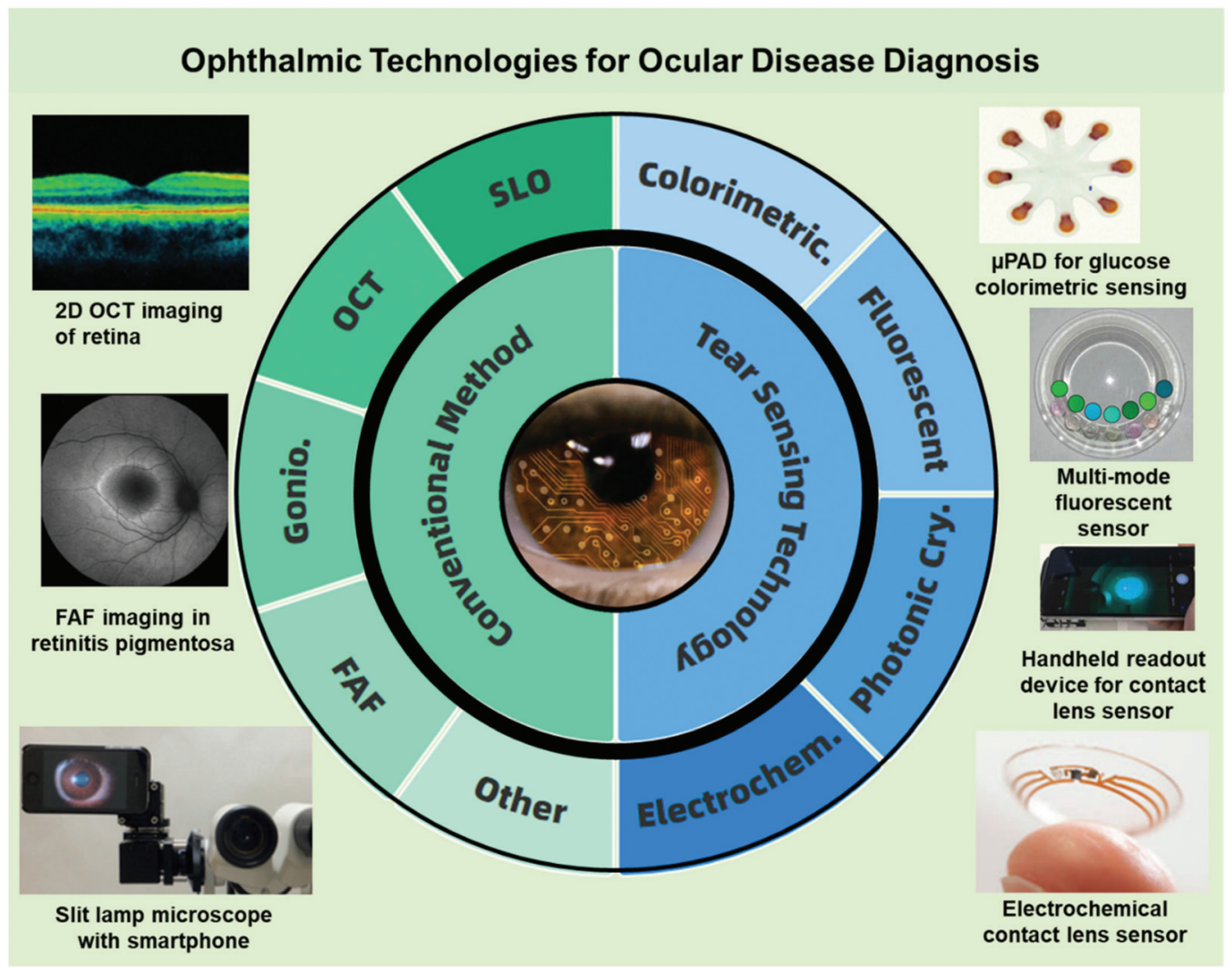

Fig. 2 Summary of ophthalmic technologies. Conventional detection methods include spectroscopies and novel imaging technologies, such as gonioscopy, slit lamp microscope, optical coherence tomography (OCT) and fundus auto-fluorescence (FAF). Four sensing methods were described in tear sensing technologies, and paper-based devices may also be applied for colorimetric sensing in the future. Both conventional and tear sensing methods can be combined with smart readout devices.

A

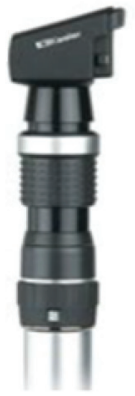

B

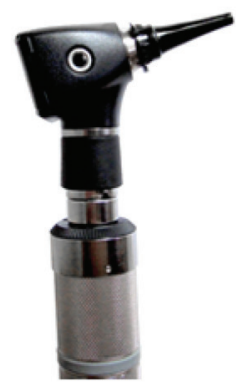

C

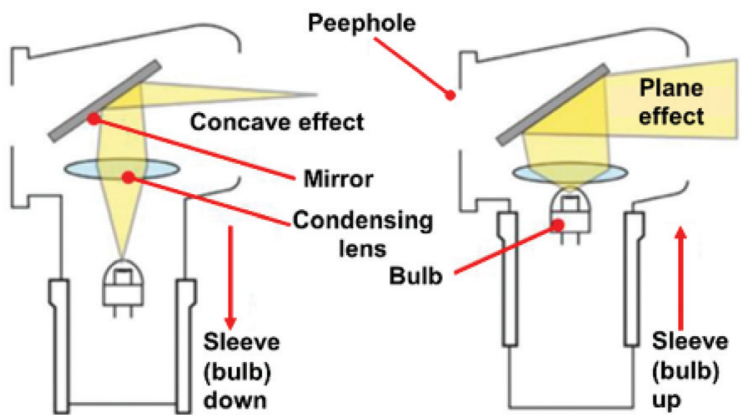

Fig. 3 Different types of retinoscopes and working scheme. (A) Streak retinoscopes: the light source produces a line or streak of light. (B) Spot retinoscope: the light source produces a spot of light. (C) Operating principles: both streak and spot retinoscopes have similar working principles, and the light can be adjusted by moving the sleeve to achieve a concave or plane effect. ${ }^{12}$ Copyright 2017 , Community Eye Health.

Gonioscopy, a widely used clinical technology, is a combination of a lens or gonioscope with an operating microscope or slit lamp. It is used to evaluate the iridocorneal angle, which is the anterior chamber angle between the cornea and iris (Fig. 5). ${ }^{13,15,17}$ The gonioscopy is an important tool for investigating, diagnosing and monitoring glaucoma disease. As one of the leading causes of irreversible blindness in the world, glaucoma has affected millions of people worldwide. ${ }^{15}$
One of the main measurement indexes in the assessment of glaucoma is an elevated IOP, which is related to internal drainage system of the eye. The underlying cause can be further investigated by using gonioscopy to measure the aforementioned iridocorneal drainage angle. This can differentiate open-angle and angle-closure glaucoma, which influences the management plan. ${ }^{14}$ However, as gonioscopy findings can be highly affected by the amount of light entering during the 
A
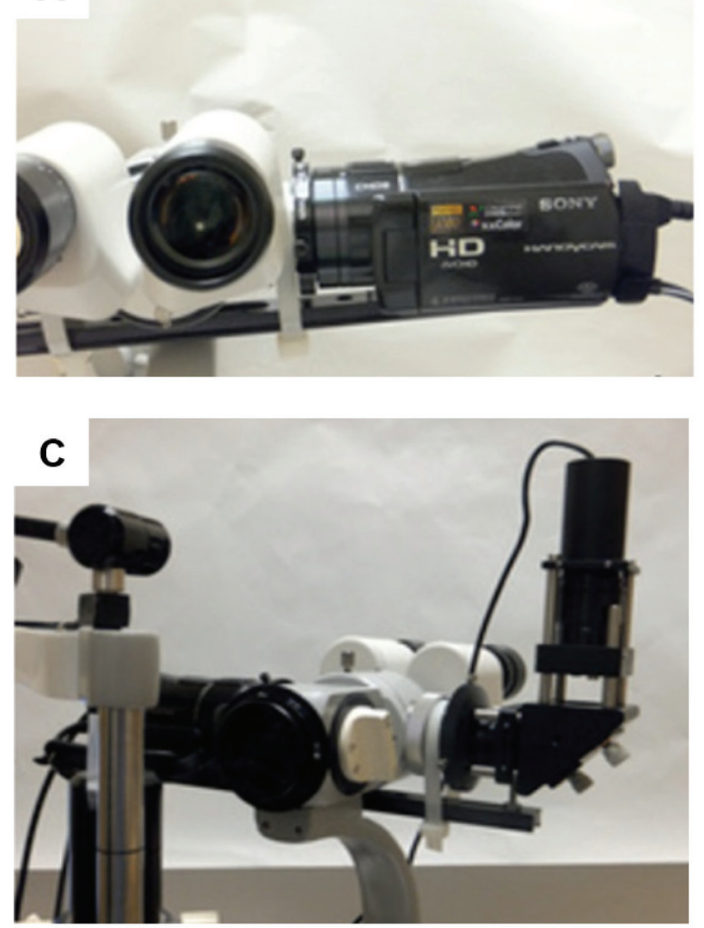

B
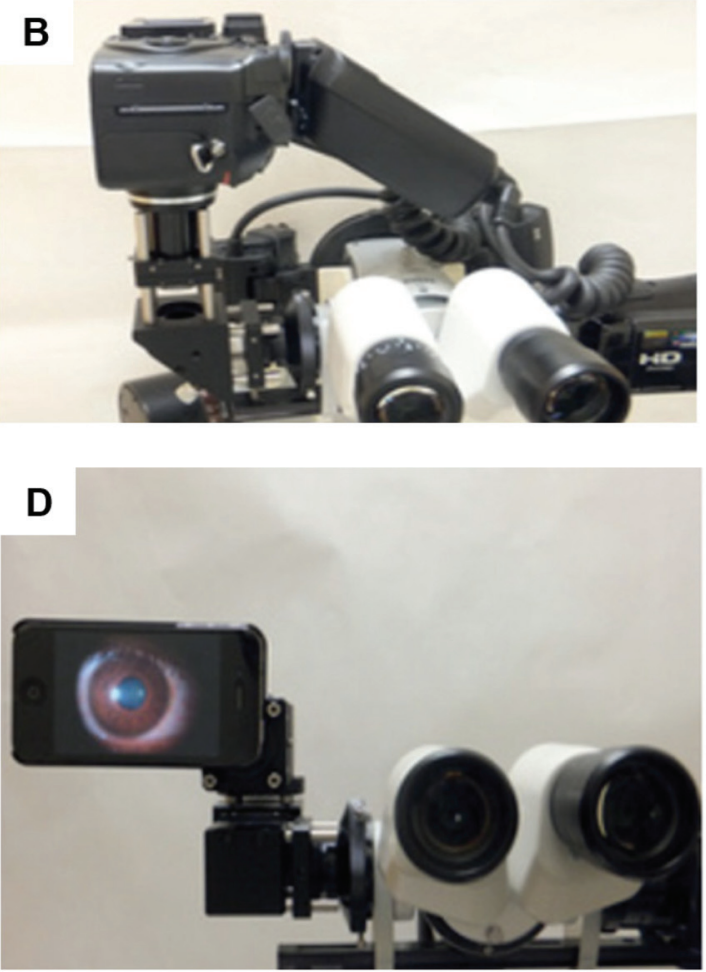

Fig. 4 External eye photography using different slit lamps. A beam splitter (50:50) was mounted on the slit lamp to adapt cameras. The Sony HDR-CX7 video camera (A) was connected to the beam splitter directly with an adaptor (digital camera adapter ring, DEC-0037; TeleVue Optics Inc., Chester, NY) without additional relay or magnification lenses in the optical pathway. On the other side of the beam splitter, a Nikon E8400 camera was connected with a relay lens (8D) and a magnification lens (slit-lamp eyepiece $\times 10$ ). A camera adapter (LNS-30DWF/CP8400; Zarf Enterprises, Spokane, WA) was used to secure the Nikon camera. A Canon MT-24EX flash (B) was attached to the slit lamp using its attachment mechanism and controlled by the Nikon camera. During the test, the Nikon camera was replaced with other cameras, including the Webcam (C), iPhone 4, iPhone 4S, and Panasonic charge-coupled device (CCD) analog camera. The iPhone 4 and 4S (D) were attached to a plate (CP4S; Thorlabs, Newton, NJ), which was secured with the relay lens. With the webcam and iPhone devices, the relay lens and magnification lens were adjusted for fine focusing. Copyright 2013, Eye \& Contact Lens: Science \& Clinical Practice.

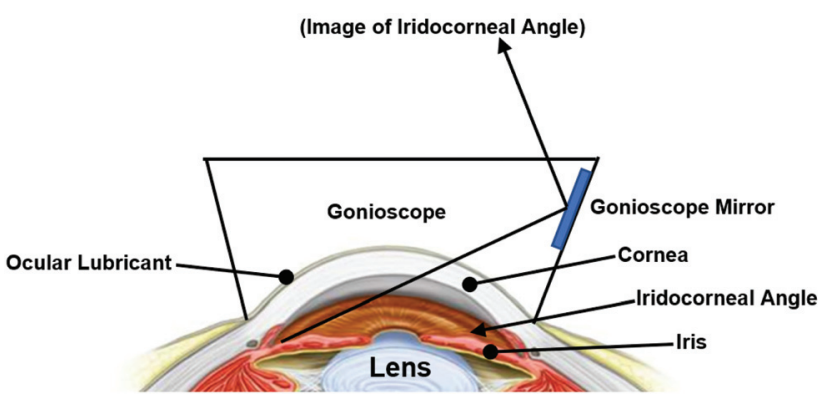

Fig. 5 Schematic iridocorneal angle. The angle is obtained by light reflection between the iris and cornea.

examination, the degree of compression on the eye by gonioscopes, examiner technique and experience, it is a subjective measurement which has low reproducibility. ${ }^{17,18}$ With the development of ophthalmic technologies, newer imaging methods have been utilized to assess the ocular angle-closure objectively. For example, ultrasound bio-microscopy with a resolution of $25 \mu \mathrm{m}-50 \mu \mathrm{m}$ provides real-time images of the drainage angle and allows visualization of posteriorly located structures, enabling detailed identification of the causes of angle closure. ${ }^{19}$ Nonetheless, these traditional measurements require the cooperation of patients, the experienced technical operation of detectors, and are normally invasive to patients. ${ }^{14}$ There is a need to explore more minimally or non-invasive imaging technologies for the diagnosis of various ocularrelated diseases.

During the late $20^{\text {th }}$ century, several technologies have been established and applied to patients with different ocular dysfunctions. These techniques have been studied and utilized for the documentation and diagnosis of ocular diseases. Various commercialized diagnostic imaging instruments are used by ophthalmologists for modern studies, including the confocal scanning laser ophthalmoscope (cSLO), the scanning laser polarimeter (GDx), and optical coherence tomography (OCT). ${ }^{15}$ OCT is one of the most widely-used noninvasive and noncontact imaging technologies in both clinical and academic researches, mainly for biomedical imaging and diagnosis. ${ }^{20,21}$ The working principle of OCT is to utilize broadband light sources with low time coherence to produce reflectivity profiles of samples with depth resolution. ${ }^{21}$ OCT is regarded as a valuable technology due to its capacity for real- 
time imaging and the usage of non-ionized near-infrared light, which is safe for repeated examination compared to other clinical tomography modalities. ${ }^{22}$ OCT is a light interference based technique. As the interference signal is received, the reflectivity profile in the beam axis can be derived. The obtained one-dimensional depth scan is named the 'A-scan'. With several neighboring 'A-scans' obtained, OCT systems can build the relevant multi-dimensional images of the target samples. ${ }^{22}$ Moreover, 'A-scans' can be obtained in two systems, which are the time-domain (TD) (Fig. 6A) and frequency domain (FD) (Fig. 6B and C). In ophthalmology, OCT systems have been applied to offer imaging of anterior and posterior segments of ocular structures directly in vivo and in vitro. ${ }^{23-25}$ In the area of ophthalmology, OCT has been acknowledged as a basic clinical tool for diagnosis and treatment monitoring in a large number of retinal diseases (Fig. 3D-F). ${ }^{21,26}$ Moreover, OCT has many other applications, including monitoring cardiac or dermatological conditions, early-stage cancer detection, intraoperative surgical guidance, and early diagnosis of musculoskeletal diseases. ${ }^{20,26-31}$ Additionally, OCT is increasingly used in the diagnosis and monitoring of age-related macular degeneration (AMD) (Fig. 3G), ${ }^{32}$ particularly in the assessment of neovascular or wet AMD. ${ }^{33-35}$

Scanning laser ophthalmoscopy (SLO) is another typical diagnostic digital imaging technology that utilizes confocal scanning laser ophthalmoscopy to obtain retinal and corneal images. SLO can provide a high spatial resolution image which is helpful for the diagnosis of glaucoma, diabetic retinopathy, and other retinal disorders. ${ }^{15,36,37}$ Compared to traditional illuminated imaging systems, the most significant advantage of SLO is the ability to acquire high-contrast images by the usage of a scanning laser source and a confocal pinhole. ${ }^{38}$ In general, the region to be imaged in SLO is raster-scanned from a focused spot over time, and the scattering lights are recorded to generate the final image. The images are initially captured and analyzed by a computer or frame grabber. However, it is difficult to monitor individual retinal cells. With the development of SLO, adaptive optics (AO) were used to compensate for monochromatic aberrations of the eye. The usage of a deformable mirror and charge-coupled device (CCD) fundus camera improved the retinal images, and made them visualize microscopic structures, such as photoreceptors in living human eye. ${ }^{38}$ The schematic operating principle of an adaptive optics scanning laser ophthalmoscopy (AOSLO) is demonstrated in Fig. 7A. With the application of this kind of SLO (Fig. 7B and C), ${ }^{39,40}$ detections in cooperation with a digital hand-held device can be developed with more accurate data for analysis.

\subsection{Imaging technology}

In recent years, various innovative methods have been developed, including ocular imaging and biosensors to monitor and diagnose ocular diseases. Fundus auto-fluorescence (FAF) is a novel imaging technique that can examine in vivo retinal conditions non-invasively. FAF imaging has been studied for

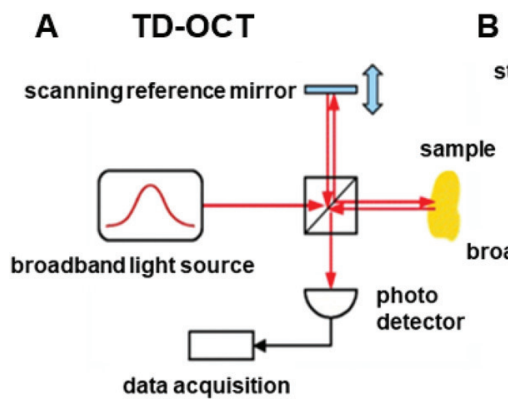

D

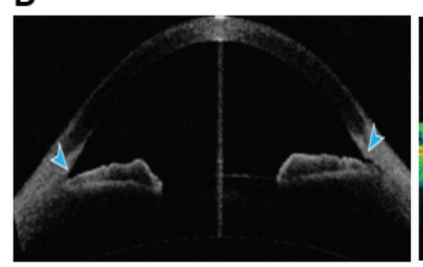

E

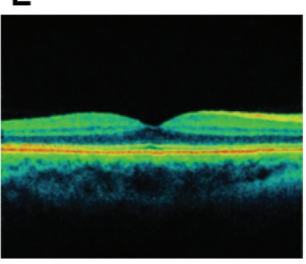

B FD-OCT (I)

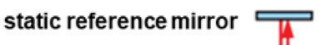

C $\quad$ FD-OCT (II)

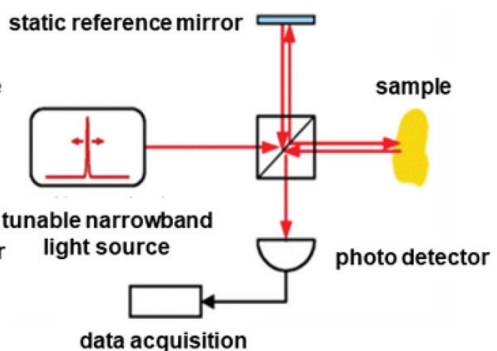

$\mathbf{F}$

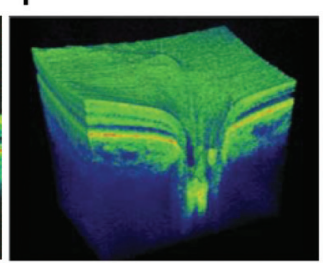

G

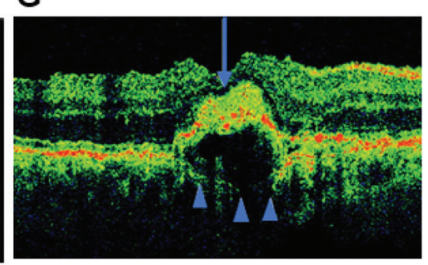

Fig. 6 OCT operating scheme and clinical images. Typically, an OCT system is based on a Michelson interferometer that directs a fraction of the light to the sample and the remaining part to a reference mirror. The back reflections from both arms interfere with a photodetector. (A) In the timedomain OCT (TD-OCT), the depth scan is performed by translating the reference mirror. (B and C) In frequency-domain OCT (FD-OCT), each depth scan is performed by sampling the interference pattern in optical frequency space with either a spectrometer and a broadband light source (I) or a photodetector and a tunable narrowband light source (II). In both cases, the reference mirror remains at a fixed position. Copyright 2011, SpringerVerlag. (D) OCT image for an open-angle anterior chamber, the arrow points to the place of the angle. (E) A cross-sectional image in two-dimensional (2D) imaging of OCT. (F) Three-dimensional (3D) image of the optic nerve head. Copyright 2014, American Medical Association. (G) A clinical image of ultra-resolution OCT for a 75 years old women with non-exudative AMD (left eye), the blue arrow indicates the penetration of moderate refractive material through RPE and lying on a pigment epithelial detachment. The blue arrowheads demonstrated the visible Brunch's membrane blow the low scattering pigment epithelial detachments. Copyright 2006, British Journal of Ophthalmology. 
A

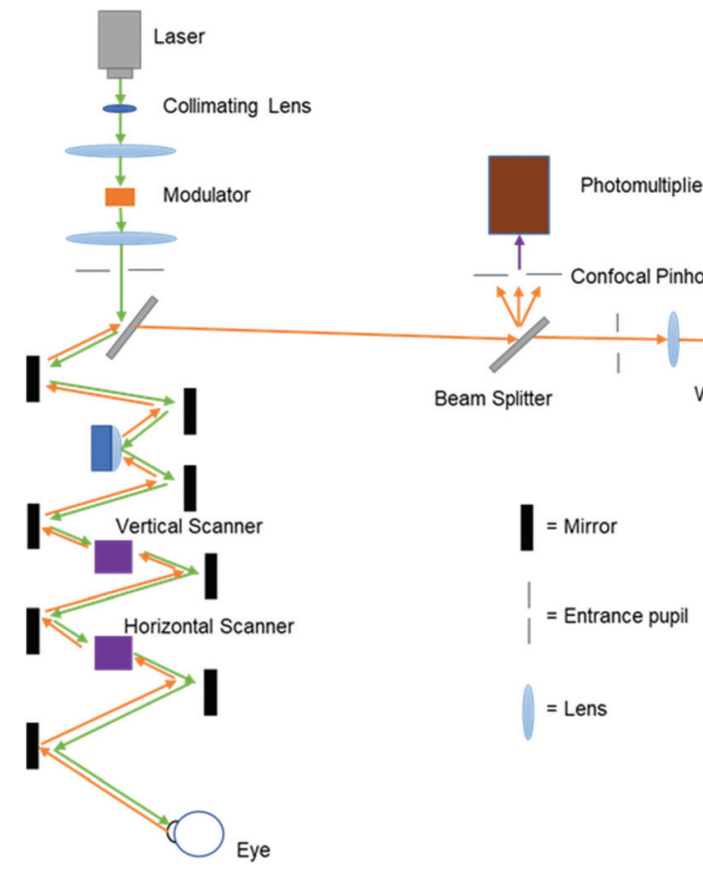

B
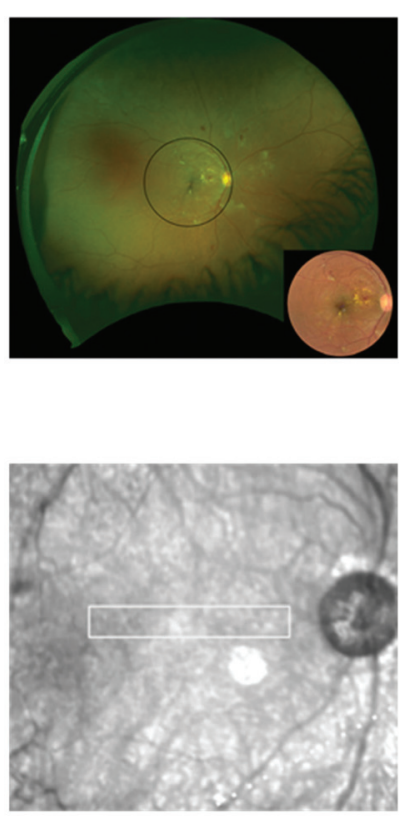

Fig. 7 Schematic set-up of AOSLO and clinical results from patients. (A) After the dilation and stabilization of pupils, a laser is shot and calibrated by a collimated lens, and reflected by a beam splitting mirror. The measuring light must pass through both horizontal and vertical mirrors during the scanning to align the moving beam to get final retinal faster images. The pathway should stay the same for the duration of detection. To diffuse optical aberrations, the light is reflected off a deformable mirror before and after the exposure of the eye. The returned laser passes onto the first beam splitter and is directed to the photomultiplier tube (PMT). The light in the photomultiplier is focused through a confocal microscopy pinhole to remove light not reflecting off of the plane of interest and is then recorded in the PMT. Light directed to the wavefront sensor array is split up by the lenslets in the array and then recorded onto a CCD camera for the detection of optical aberrations. These aberrations are then subtracted from the images recorded in the PMT to vastly increase lateral resolution. Copyright 2002, Optical Society of America. (B) The resulted figure was taken from SLO and then used Image $\mathrm{J}$ to crop into the equivalent size and 45-degree of position of the photograph from diabetic patients. Copyright Acta Ophthalmologic, 2019. (C) The AO-SLO image from a right eye of patient with diabetic retinopathy. Copyright International Journal of Ophthalmology, 2017.

the past decade, and has been indicated as an efficient clinical research tool for diagnosing ocular diseases, understanding their pathophysiological mechanisms, identifying predictive markers for disease progresses, and monitoring related therapies. The main sources of fluorophores on the retina are lipofuscin and melano-lipofuscin which are endogenous. This is advantageous for FAF imaging of the retina, as it does not require any injection of dyes. Therefore, FAF imaging permits topographic mapping of the distribution of various fluorophores that may appear with disease in the outer retina and sub-neurosensory space, including lipofuscin in the retinal pigment epithelium (RPE) cell monolayer and photoreceptors. ${ }^{41}$ The excessive accumulation or depletion of fluorophores within RPE cells and retinal tissues can lead to common downstream pathogenetic pathways in various hereditary and complicated retinal diseases. The RPE, a single layer of polygonal cells between the choroid and neurosensory retina, has an important role for normal retinal functioning. It maintains the photoreceptor renewal process by phagocytosing and degrading lysosomes of the pigmented outer sections of photoreceptors. Over 3 billion of these outer segments will be phagocytosed by one RPE cell during its lifetime. The accumu- lation of lipofuscin occurs with the aging of post-mitotic RPE cells; these segments are incompletely or partially broken down. One of the major components of lipofuscin is $N$-retinyl$\mathrm{N}$-retinylidene ethanolamine (A2E) (Fig. 8A), and the accumulation of this degraded $\mathrm{A} 2 \mathrm{E}$ in the lysosomal compartment of RPE cells is regarded a symbol of aging in the eyes. ${ }^{42}$ The excess deposition of lipofuscin is claimed to be pathologic and is related to a certain degree of blindness. Another component of lipofuscin is all-trans-retinal, which is a toxic aldehyde. As the excess of all-trans-retinal exposes to the light, A2E can be produced in the outer section of photoreceptors (Fig. 8B). Therefore, with the application of FAF technology, the variation of integrity and metabolism of retinal cells can be easily analyzed. Other ocular fluorophores and pigments can also be analyzed under FAF technology, including lipofuscin, melanin, and rhodopsin. ${ }^{42}$

FAF images are commonly obtained by the use of confocal scanning laser ophthalmoscopy (cSLO) (Fig. 9). As for adopting the confocal optics, the FAF technique can overcome the disturbance caused by the auto-fluorescence of preretinal structures such as the lens. Confocal optics keep the reflectance of scanning laser and the retinal fluorescence developing at the 

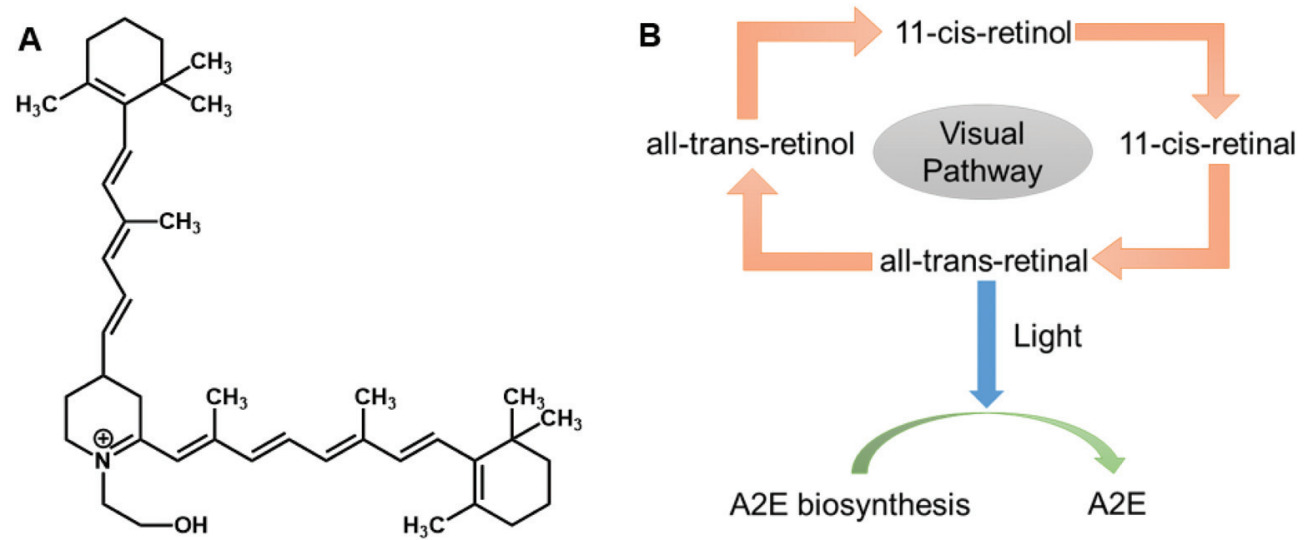

Fig. 8 Demonstrations for FAF technique. (A) Chemical Structure of $N$-retinyl- $N$-retinylidene ethanolamine (A2E). This is a major component of lipofuscin that will not be recognized or completely decomposed by lysosomal enzymes, leading to an increase in its concentration in lysosomes. The accumulation of this degraded A2E in the lysosomal compartment of RPE cells is a symbol of aging in the eyes. (B) Biochemical pathway of lipofuscin. Photoreceptors cannot convert all-trans-retinal back into 11-cis-retinal after transduction of light energy into electrical impulses when lacking cis-trans retinal isomerase function. Due to this, the unconsumed all-trans-retinal will accumulate in the photoreceptor and form bisretinoids that contribute to lipofuscin production by oxidation.
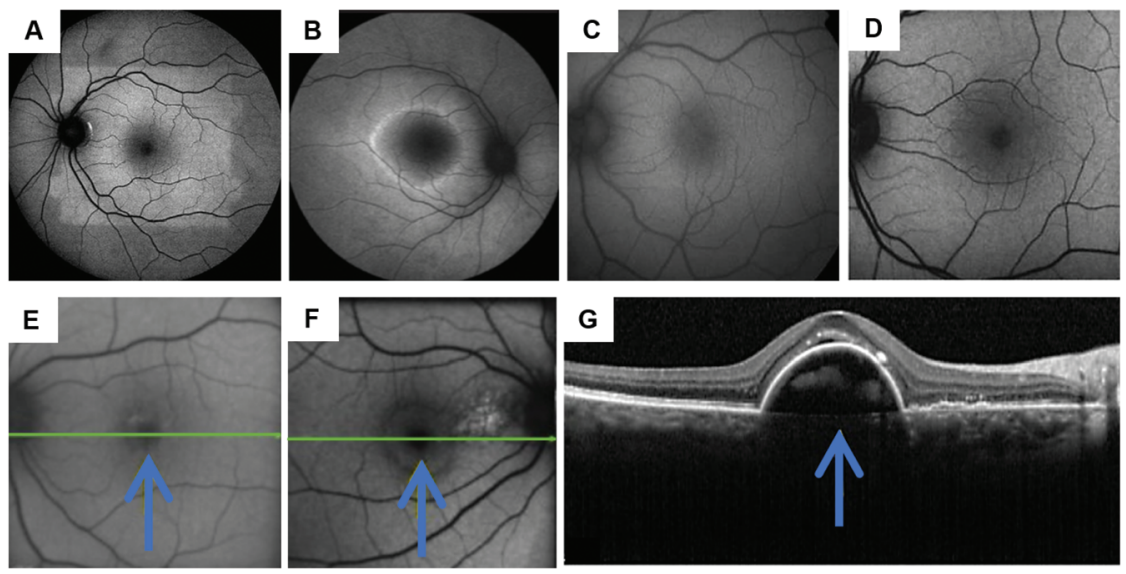

Fig. 9 FAF images of healthy and diseased retinas. (A) Bleaching effect of rhodopsin under 50 degree of FAF; visual pigments can absorb the excitation beam and decrease autofluorescence. ${ }^{43}$ (B) Short-wavelength-autofluorescence (AF) image showing a ring-shaped AF in retinitis pigmentosa. (C) Image of normal, healthy retina under FAF using a fundus camera accompanied by a Spaide filter. (D) Image of normal, healthy retina under FAF via cSLO. Copyright 2016, Springer Nature. (E) A photograph of a diffusing area of the fovea and surrounding area in an eye under hypo-autofluorescence. It is a diseased eye with cystic macular oedema with vein occlusion in central retina. The blue arrow in the image is pointing towards the area of hypo-autofluorescence. (F) Neovascular AMD, right eye. (G) The OCT image indicates the site of neovascularization has fovea surrounded by a band of hypo-autofluorescence. Arrows indicate the direction of the disease process surrounding the fovea. Copyright 2014, Elsevier.

same optical plane. For a standard ophthalmoscope, the excitation and emission filter wavelengths are $488 \mathrm{~nm}$ and 500-529 nm respectively, which stay in the range of blue light. Thus, the cSLO-AF is also named blue-AF or short-wavelength (SW)-AF. A longer excitation wavelength $(790 \mathrm{~nm})$ can be obtained for near infra-red (NIR)-AF with confocal optics as well. Near-AF has a higher emission filter (>800 nm) compare to cSLO-AF, but the visible signal is $60-100$ times weaker than that in blue light. However, the major fluorophore in NIR-AF is melanin, so the fluorescence is more intense in choroidal tissues and RPE cells where the melanin is denser. Moreover, a fundus camera can also be accommodated to supply FAF images by projecting a single flashlight on the entire retina at one time. Therefore, the auto-fluorescence can still be elicited from pre-retinal structures without a confocal optics system. The utilization of typical longer wavelengths, for example with an excitation filter between $535-580 \mathrm{~nm}$ and barrier filter between 615-715 $\mathrm{nm}$, can overcome the potential interferences. This type of FAF technique has been developed in recent years and is named green $\mathrm{AF}^{41}$ The intensity of each pixel signal with different spatial distribution can be observed in FAF images with a range of 0-255 (gray values). A low pixel value with dark color indicates a low intensity, whereas a high pixel value with bright color indicates a high intensity. The accretion of auto-fluorescence materials and deposits in RPE cells in retinal dystrophies has been reported in various histo- 
pathological studies. Any deviation from a normal recording would be recognized when analyzing the FAF image. Therefore, FAF imaging technologies have been applied to study a wide range of ocular diseases, including retinitis pigmentosa, diabetic retinopathy, white dot syndromes, retinal artery occlusion, and age-related macular degeneration (AMD) (Fig. 9). Moreover, the obtained FAF image can be analyzed together with the cross-sectional OCT images.

Hence, FAF imaging technology can be significant in providing informative data for retinal dystrophies, where the health of RPE cells is an important measurement index. The variation of lipofuscin in RPE cells can be visualized through images. Furthermore, the imaging technique can also have potential future applications for identifying the highrisk characteristics or biomarkers of ocular diseases and performing clinical trial interventions in specific cases. The combination of the fluorescence and spectroscopy could provide creative ideas for ocular smart sensing technologies.

\section{Contact lens sensors}

\subsection{Advances in contact lens sensors}

Contact lenses, as wearable devices for various ocular purposes, are used by over 150 million people globally ${ }^{44}$ and 3.7 million people in the United Kingdom. ${ }^{45}$ The current applications of contact lenses include vision correction, therapeutics, and cosmetics. ${ }^{46}$ In recent years, contact lenses are increasingly being developed as minimally invasive continuous sensing platforms for diagnostics and drug delivery in ophthalmology. ${ }^{44}$ The design and materials of a contact lens are based on their applied purpose. The structure of tear film is altered after the insertion of contact lens onto the ocular. The evaporation rate of tear film would increase due to the disruption of lens within tear film and lead to the lipid layer discontinuous. ${ }^{47}$ All contact lenses can alter the tear film as they interact with the components within tears and interfere with the lid-corneal congruity, hence it can be divided into three categories due to their physiochemical properties and design characteristics, including rigid lenses, elastomeric lenses and hydrogel lenses. Different types of contact lenses react differently with tear film, it can be varied due to the hydration, thickness, speed of dehydration and evaporation rate. ${ }^{47,48}$ Moreover, contact lenses are also defined by their geometry, and can be classified as scleral, mini-scleral, or corneal (Fig. 10A).

Over the last decade, contact lens sensors have been widely studied, with the monitoring of ocular disorders, such as dry eye, glaucoma, and diabetic retinopathy, being one of their primary applications. ${ }^{44}$ Various indicators have been studied via contact lens sensors including $\mathrm{pH}$, temperature, electrolytes, and IOP. ${ }^{46,49-52}$ However, tear fluid analysis has not been comprehensively studied. Tear film comprises of three layers: the lipid layer, aqueous layer, and mucous layer. ${ }^{53}$ Research involving proteomic analyses reveals that over 2000 proteins are found in tears. ${ }^{54}$ Established functions of the tear film include creating a smooth surface, supplying sufficient oxygen and nutrients to the ocular surface, removing irritants, and assisting the immune system on the ocular surface. ${ }^{55}$ Due to the less complex nature of tear fluid than serum and plasma, numerous biomarkers in tear fluid may be useful in predictive, preventive, and personalized medicine. ${ }^{54}$ In addition, contact lenses can also be used to assist typical ocular dysfunctions and color vision deficiency (CVD). Recently, researchers have developed contact lenses that cross-link with selected dyes or nanoparticles to block out the wavelengths $(540-580 \mathrm{~nm})$ that create issues for people with red-green CVD. ${ }^{56,57}$ Overall, contact lenses involved in continuous tear film sampling, and some functionalized contact lens sensors have many potential applications for disease diagnostics and treatment.

Contact lenses are utilized globally for the correction of refractive errors, such as myopia, hyperopia, and astigmatism. ${ }^{44}$ Therapeutic contact lenses can be used to maintain and restore the integrity of ocular tissues, such as in corneal abrasion. ${ }^{44,58}$ Furthermore, cosmetic contact lenses are widely available, for example coloured lenses are extremely popular in Asian countries. ${ }^{44}$ Contact lenses developed as smart devices for continuous monitoring in POC settings have received increasing attention recently, because outcome sensors can be powered externally and accompanied with wireless smartphone readouts. $^{44,49-51}$

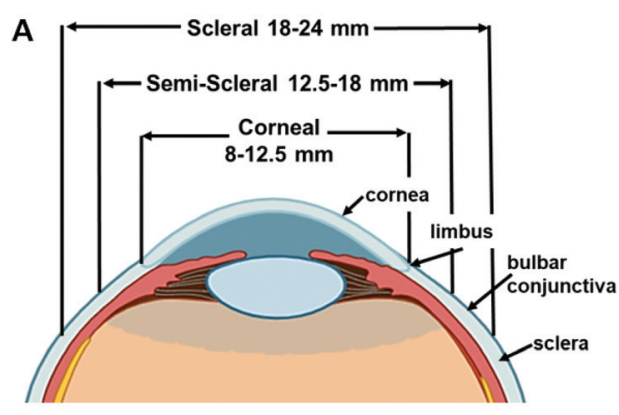

B

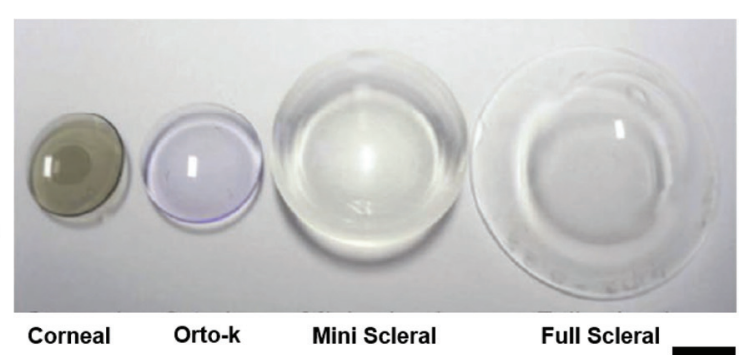

Fig. 10 The contact lenses species. (A) The variety of contact lenses is indicated with their diameters. (B) Image of different contact lenses. Scale bar: $4.0 \mathrm{~mm}$ Copyright 2017, Elsevier. 
Although contact lenses offer convenience to users, there are disadvantages to their use. ${ }^{59}$ One common complaint with contact lens use is a feeling of discomfort. ${ }^{60}$ Other adverse effects include allergic reactions, microbial keratitis, and corneal inflammation. ${ }^{44,61}$ It has been studied that $40-60 \%$ of contact lens users worldwide with any form of allergy have ocular symptoms. ${ }^{61}$

\subsection{Sensing technology and applications}

Tear films are a complicated exocrine fluid responsible for cleaning and lubricating eyes. As mentioned previously, outer lipid, aqueous, and inner mucin form the three layers of tear films, and each layer has different functions and compositions. ${ }^{46}$ The barrier between the blood contained within vasculature, and tear fluid, causes the differences in composition between the blood and tear fluid. However, certain metabolic processes occurring during plasma leakage can allow the transfer of blood components through the blood-tear barrier into tears, which builds a close relationship between blood and tear fluid. ${ }^{46,62}$ With a wide potential detection range in tear fluid, it has been considered an innovative research platform for the diagnosis of ocular diseases, as well as for ocular abnormalities induced by other diseases, such as diabetes, cancer, and neurological disorders. ${ }^{11}$ There have been various biomarkers studied for their potential use as indicators for monitoring and diagnosing diseases (Table 1). Hence, the integration of specific biomarkers in contact lens sensors creates the possibility of diagnosing and monitoring the progression of certain diseases.

Contact lens sensors are utilized for monitoring and transferring distributive information of various analytes in tear fluid into signals. These signals can be obtained optically by an instrument or an observer. Some diseases may not require a continuous monitoring platform, in which case contact lens sensors can also serve as a one-time diagnostic tool. In this circumstance, the contact lenses can accumulate tear fluids during wear, and this can be analyzed when the lenses are taken off. There have been various emerging methods studied based on contact lens sensors, including colorimetric, fluo- rescence, electrochemical, and photonic crystal sensing. The performance of different contact lens sensors can vary due to conditions (Table 2), such as the detection range and response time.

3.2.1. Colorimetric sensing. Colorimetric sensing is developed from colorimetric assays. It involves the use of a color reagent under a specific wavelength of light to measure the concentration of a target chemical element or compound in a solution. It can be used for both organic and inorganic compounds, and the use of enzymatic chemicals is flexible. The measurement is based on the absorption of photons of light within the visible spectrum with wavelengths between $400 \mathrm{~nm}$ and $800 \mathrm{~nm} \cdot{ }^{107}$ It is a photometric technique developed from the fundamental Beer-Lambert Law (eqn (1)). ${ }^{108}$ When a beam of incident light with an intensity of $I_{0}$ passes through a solution, the light will transfer in three ways including reflection, absorption, and transmission (eqn (2)).

$$
\begin{gathered}
A=\log _{10} \frac{I_{0}}{I_{\mathrm{t}}}=a b c \\
I_{0}=I_{\mathrm{r}}+I_{\mathrm{a}}+I_{\mathrm{t}}
\end{gathered}
$$

where $I_{0}$ is the intensity of light; $I_{\mathrm{r}}$ is the reflected light; $I_{\mathrm{a}}$ is the absorbed light; $I_{\mathrm{t}}$ is the transmitted light; $A$ indicates the absorbance (optical density); $a$ is the absorptivity (extinction coefficient); $b$ is the optical path length and $c$ is the concentration of the solution. The amount of reflected light $\left(I_{\mathrm{r}}\right)$, that acts as a controlled variable, will be eliminated when measuring $I_{0}$ and $I_{\mathrm{t}}$, thus allowing the amount of absorbed light to be determined. Therefore, the variation of solution concentrations is proportional to the detected absorbance $(A)$ and accompanied by a series of colour changes. Sometimes the absorption spectra can achieve a wider range of detection, such as UV-vis spectra (200-800 nm). In the visible region of the spectrum, the consisted photon energies stay at around 36-72 $\mathrm{kcal} \mathrm{mol}^{-1}$, whereas in the near-ultraviolet region that is used for UV-vis spectroscopic observation in some sensing processes, the spectral scale is extended to $200 \mathrm{~nm}$ with the energy of $143 \mathrm{kcal} \mathrm{mol}^{-1}$. $^{107}$

\begin{tabular}{|c|c|c|}
\hline Disease/conditions & Biomarkers & Ref. \\
\hline Dry eye disease & LCN-1; IL-1; IL-6; IL-22; MMP-9 & $\begin{array}{l}63,64,65,66,67,68 \text { and } \\
69\end{array}$ \\
\hline Ocular allergy & Hemopexin; Substance P, CGRP, VIP; Transferrin, Mammaglobin B; IgE abs & $70,71,72,73$ and 74 \\
\hline Keratoconus & MMP-9, IL-6; IL-1 $\beta$, IFN- $\gamma ;$ SFRP-1; Prolidase & $75,76,77$ and 78 \\
\hline Trachoma & IgG against cHSP60, CPAF and CT975 & 79 \\
\hline Glaucoma & $\begin{array}{l}\text { IL-12p70; Acetyl carnitine (C2); IFN- } \gamma \text {; GM-CSF; IL-5; carbonic anhydrase, lipase and } \\
\text { antioxidants; BDNF }\end{array}$ & $80,81,82,83$ and 84 \\
\hline Aniridia & Zinc- $\alpha 2$-glycopritein, lactoferrin, VEGF; Ap4A and Ap5A & 85 and 86 \\
\hline Multiple sclerosis & Ser, Asp, His & 87 \\
\hline Cancer & Cystatin SA; protein S100-A4; Keratin (type II) etc. & 88 \\
\hline $\begin{array}{l}\text { Diabetic } \\
\text { retinopathy }\end{array}$ & Lysozyme C; lacritin, lipophilin A, Ig lambda chain; TNF- $\alpha$; haemoglobin A1c (HbA1c) & $89,90,91$ and 92 \\
\hline System sclerosis & CFD; CHI3L1; CRP; EGF; IP-10, MCP-1; MIG; MMP-9; VDBP & 93 and 94 \\
\hline $\begin{array}{l}\text { Parkinson's } \\
\text { disease }\end{array}$ & TFN- $\alpha$; alpha-1 antichymotrypsin & 95 and 96 \\
\hline
\end{tabular}

Table 1 Biomarkers for ocular diseases 
Table 2 Performances on different contact lens sensors

\begin{tabular}{|c|c|c|c|c|c|}
\hline Method & Range & $\begin{array}{l}\text { Readout } \\
\text { time }\end{array}$ & Analyte & Power & Ref. \\
\hline \multirow[t]{5}{*}{ Colorimetric } & $5-8$ & $10 \mathrm{~s}$ & $\mathrm{pH}$ & No & $\begin{array}{l}50,97 \\
\text { and } 98\end{array}$ \\
\hline & 0-10 mM & $15 \mathrm{~s}$ & Glucose & No & 97 and \\
\hline & $0-160 \mu \mathrm{M}$ & & Nitrite & No & 98 \\
\hline & $0-8 \mathrm{mg} \mathrm{mL}^{-1}$ & $15 \mathrm{~s}$ & $\begin{array}{l}\text { Protein (bovine serum } \\
\text { albumin (BSA)) }\end{array}$ & No & \\
\hline & $50-300 \mathrm{mg} \mathrm{L}^{-1}$ & $25 \mathrm{~s}$ & Ascorbic acid & No & 98 \\
\hline \multirow[t]{3}{*}{ Fluorescence } & $50 \mu \mathrm{M}-100 \mathrm{mM}$ & $<45 \min$ & Glucose & No & 99 \\
\hline & $7-8$ & - & $\mathrm{pH}$ & No & 100 \\
\hline & $\begin{array}{l}0-100 \mathrm{mM} \mathrm{Na}^{+} ; 0-220 \mathrm{mM} \mathrm{Cl}^{-} ; 0-50 \mathrm{mM} \mathrm{K}^{+} ; 0.5-1.25 \mathrm{mM} \\
\mathrm{Ca}^{2+} ; 0.5-0.8 \mathrm{mM} \mathrm{Mg}^{2+} ; 10-20 \mu \mathrm{M} \mathrm{Zn}^{2+}\end{array}$ & & $\begin{array}{l}\text { Ions }\left(\mathrm{Na}^{+}, \mathrm{Cl}^{-}, \mathrm{K}^{+}, \mathrm{Ca}^{2+}\right. \\
\left.\mathrm{Mg}^{2+} \text {, and } \mathrm{Zn}^{2+}\right)\end{array}$ & & \\
\hline \multirow[t]{3}{*}{ Photonic Crystal } & $0-50 \mathrm{mM}$ & $<30 \min$ & Glucose & No & 101 \\
\hline & $0-50 \mathrm{mM}$ & $3 \mathrm{~min}$ & Glucose & No & 102 \\
\hline & $0-50 \mathrm{mM}$ & $30 \mathrm{~min}$ & Glucose & No & 103 \\
\hline \multirow[t]{3}{*}{ Electrochemistry } & $0-2 \mathrm{mM}$ & $<100 \mathrm{~s}$ & Glucose & Yes & 104 \\
\hline & $50 \mu \mathrm{M}-5 \mathrm{mM}$ & $<8 \mathrm{~s}$ & Glucose & Yes & 105 \\
\hline & $50 \mu \mathrm{M}-5 \mathrm{mM}$ & $35 \mathrm{~s}$ & L-Lactate & Yes & 106 \\
\hline
\end{tabular}

Recently, colorimetric sensing has received attention in contact lens sensor design for its specificity, sensitivity, and feasibility. Colorimetry theory has been used to study a wide range of objects, such as $\mathrm{pH}$, electrolytes, and some proteins. One type of anthocyanin-contained contact lens sensor was developed for monitoring the $\mathrm{pH}$ level in tear fluid with the aid of RGB triplets colour model data collection. As a natural bioactive water-soluble compound, anthocyanins have been studied as potential indicators for $\mathrm{pH}$ sensing in tears. The chemical structure of anthocyanins is modified when the concentration of hydrogen ions varies (Fig. 11A), and fabricated sensors are expected to provide a variation of color with changes of $\mathrm{pH}$ in the tear film. Therefore, the anthocyaninfunctionalized contact lenses were constructed as wearable sensors to monitor the ocular $\mathrm{pH}$ within the range of $6.5-7.5 .^{50}$ In this experiment, varying dye concentrations were diluted in deionized water with different percentages and converted as a related objective to functionalize the contact lens sensors. Moreover, the RGB color model was utilized to obtain quantitative values for comparisons with naturally extracted dye and with delphinidin chloride dye in an aqueous solution from 0.0 to $1.5 \mathrm{mM} .^{50}$ Furthermore, the soaking time and the leakage of dye were also studied as important criteria for evaluating the functions of contact lens sensors. As a result, the best accepted time for soaking was $24 \mathrm{~h}$ and the dye leakage that happened in deionized water after $18 \mathrm{~h}$ was considered negligible. During the fabrication of contact lens $\mathrm{pH}$ sensors, the soft contact lenses were selected because of their relatively higher hydrophilicity. The sensors were gained from the impregnation of anthocyanin solution with polymeric matrices of commercial soft contact lenses under soaking and dropcasting that processed on either the concave or the convex face of contact lenses. ${ }^{50}$ As a result, the drop-casting method contributed to the limited and heterogenous distribution of dye, but the soaking process provided a higher permeation and well-proportioned spatial distribution of dye. To obtain an efficient colorimetric detection of hydrogen ions, contact lenses with and without lacreon were evaluated. Lacreon-containing contact lenses were selected for reporting colour gradients easily distinguished by the naked eye. The spectral performance was optimized in a transmission mode setup (Fig. 11B), and a decreasing trend was observed under $500-650 \mathrm{~nm}$ band and after the peak at $660 \mathrm{~nm}$ with the concentration of dye ranging from $0.3 \mathrm{mM}$ to $1.5 \mathrm{mM}$ under the $\mathrm{pH}$ of 6.5, 7.0, and 7.5 (Fig. 11C-E). Moreover, an alternative method for constructing $\mathrm{pH}$-sensitive contact lenses was tested using photopolymerization to cross-link the anthocyanin dye with poly-HEMA soft contact lenses. However, the color shift from pH 6.5 to 7.4 was not significant enough for observation by naked eye. Other hydrogel matrices including polyvinyl alcohol and polyacrylamide were proposed. ${ }^{50}$

Microfluidic contact lens sensors, with multiple sensing channels, have been developed to detect multiple analytes in tear fluid including $\mathrm{pH}$, glucose, nitrite, proteins, and ascorbic acid. ${ }^{97,98,152}$ The microfluidic patterns can be fabricated through $\mathrm{CO}_{2}$ laser ablation to provide multi-channel concaves (Fig. $11 \mathrm{~F}(\mathrm{a}))$ within the contact lens for monitoring targeted analytes (Fig. $11 \mathrm{~F}(\mathrm{~b})$ ). Three layers were attached onto the contact lens: a paper substrate, an adhesive layer, and a sensing pad. As a result, the sensor exhibited sensitivities at $12.23 \mathrm{~nm}$ per $\mathrm{pH}$ unit, $1.4 \mathrm{~nm} \mathrm{mmol}{ }^{-1} \mathrm{~L}^{-1}$ of glucose, $0.49 \mathrm{~nm}$ $\mathrm{g}^{-1} \mathrm{~L}^{-1}$ of proteins, $0.03 \mathrm{~nm} \mu \mathrm{mol}^{-1} \mathrm{~L}^{-1}$ of nitrites, and $0.05 \mathrm{~nm} \mathrm{mg}^{-1} \mathrm{~L}^{-1}$ of ascorbic acid. The ascorbic acid channel provided the longest response time of $25 \mathrm{~s}^{98}$ and the other channels had response times within a range of $15 \mathrm{s.}^{97}$ Furthermore, colorimetric readouts of this contact lens sensor were obtained from the smartphone-MATLAB algorithm (Fig. $11 \mathrm{~F}(\mathrm{c})$ ) which offers convenience at the POC platform. The paper-based sensing that was utilized during this research can also be applied for other biomarkers within tear fluid. ${ }^{11}$

Though not fully assessed yet with contact lens sensors, a large number of paper-based sensing experiments been carried out on tear analytes for colorimetric sensing, such as glucose, immunoglobin $\mathrm{G}(\mathrm{Ig} \mathrm{G})$, and brain-derived neurotropic 

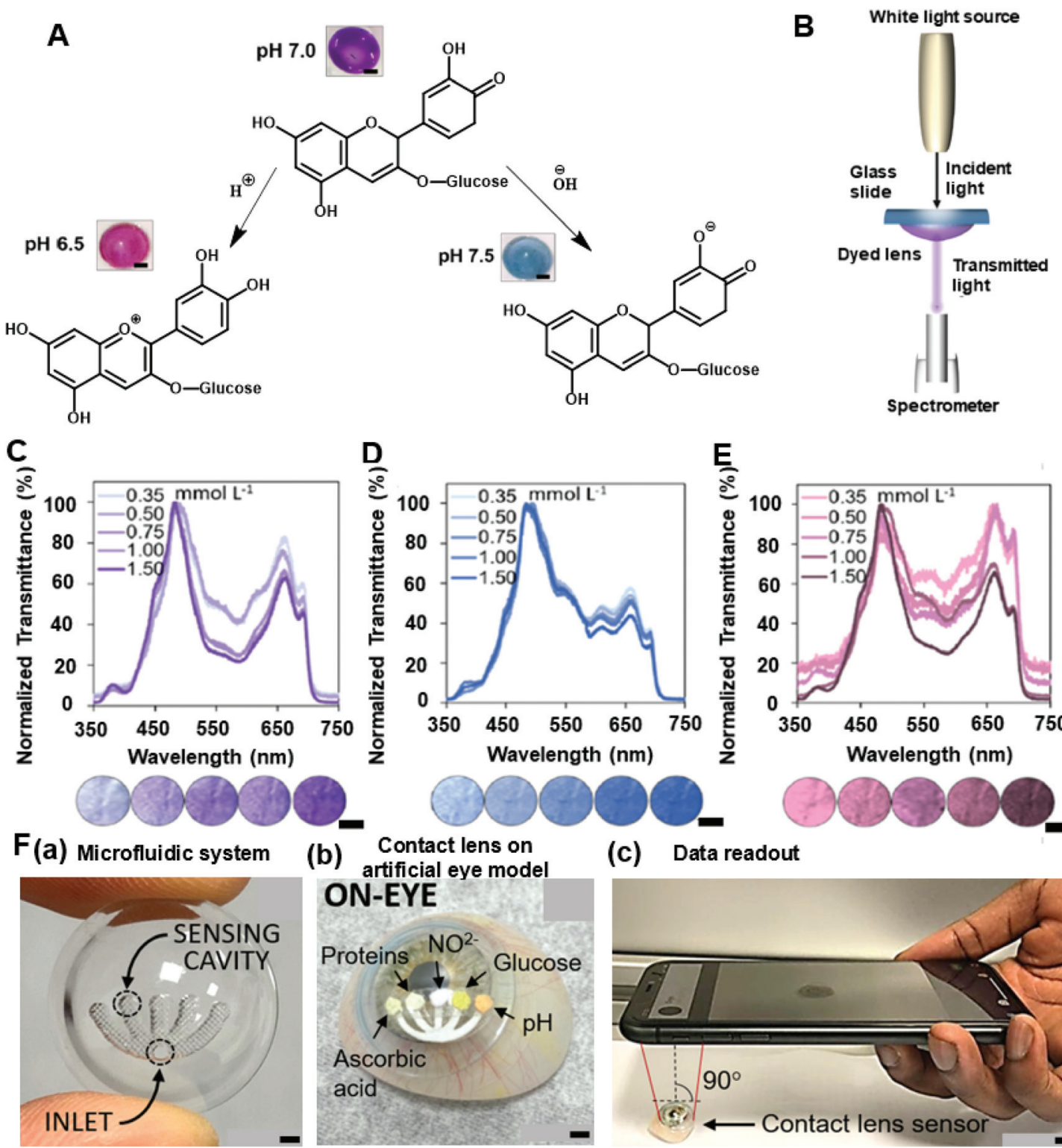
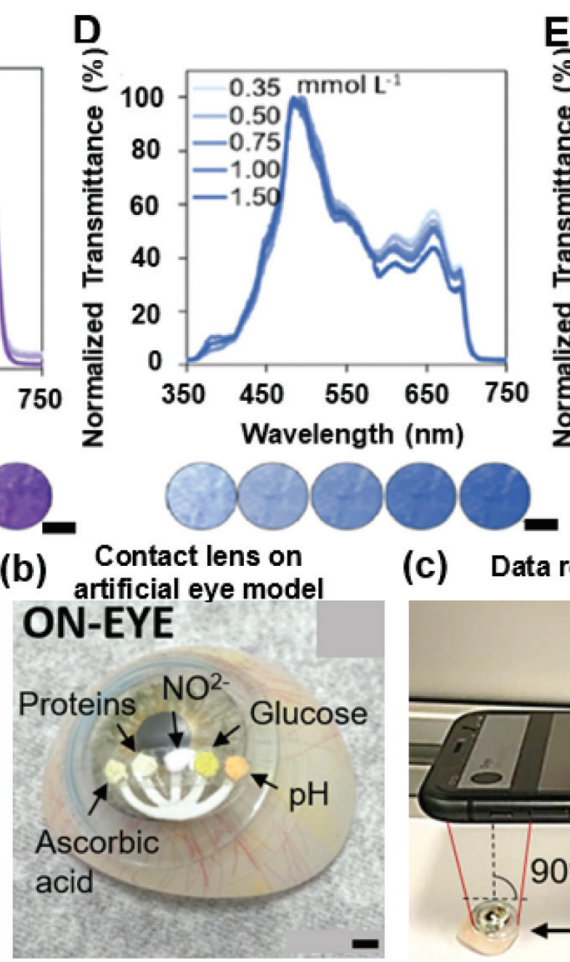

(c) Data readout
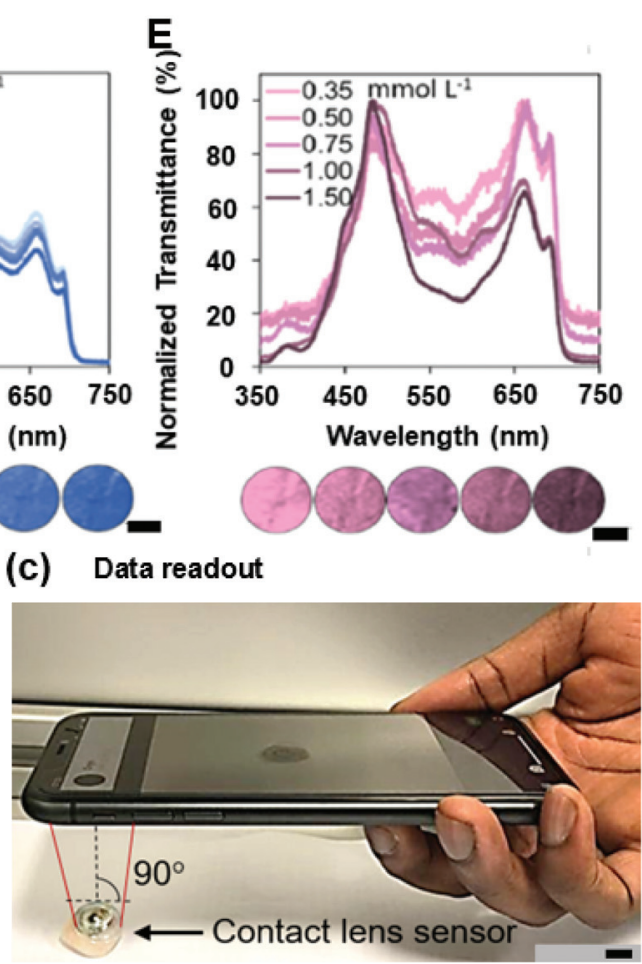

Fig. 11 The relative theory and data obtained from the contact lens sensor for pH monitoring. (A) The variation of anthocyanin chemical structure based on different hydrogen ion concentrations. Under acidic conditions ( $\mathrm{pH} 6.5)$, the contact lens sensor showed a pink colour, and the colour shifted to blue when the solution changed to alkaline conditions ( $\mathrm{pH}$ 7.5). (B) The scheme for the setup of spectroscopy measurement in the transmission mode. Using a white light source as the incident light which passes through the glass slide and exposes on the fabricated dyed contact lens, the transmitted light is analysed by the spectrometer with a level of 200 lux. (C) Transmittance spectra result under pH 6.5 within a concentration of anthocyanin range of $0.35-1.50 \mathrm{mM}$. (D) Transmittance spectra result under $\mathrm{pH} 7.0(0.35-1.5 \mathrm{mM}$ of anthocyanin). (E) Under an alkaline (pH 7.5) condition, the resulted transmittance spectra. According to the results of the experiments, the concentration of dye in the solution indicated a function of transmittance. In the range of 500-650 nm, a decreasing trend was observed and showed the peak at $660 \mathrm{~nm}$ with the increased concentration of dye. Scale bar: $2.0 \mathrm{~mm}$. Copyright 2019, American Chemical Society. (F) Schematics of microfluidic contact lens sensor. (a) A microfluidic system was fabricated through $\mathrm{CO}_{2}$ laser ablation which consisted of inlet cave and sensing caves. (b) The final contact lens sensor on an artificial eye model with five different analytes: $\mathrm{pH}$; glucose; nitrite; proteins and ascorbic acid; scale bar: $1.5 \mathrm{~cm}$. (c) A simulation photograph of the working process on the ocular surface for data readout using a mobile phone. Scale bar: $2 \mathrm{~cm}$. Copyright 2020, Lab on a Chip.

factors (BDNF), to indicate and monitor ocular diseases and other forms of ocular dysfunction. One colorimetric sensing test of glucose and uric acid (UA) in tears was developed using chitosan-modified microfluidic paper-based analytical devices ( $\mu$ PAD). ${ }^{109}$ The best colorimetric sensitivity for glucose and UA was obtained with a linear response in a concentration range of 0.1-1.0 $\mathrm{mM}$ with the aid of a chromogenic solution, consisting of 4-amino anti-pyrine and sodium 3,5-dichloro-2-hydroxybenzenesulfonate (4-AAP/DHBS). The $\mu$ PADs were fabricated by a stamping process. ${ }^{110}$ There are eight detection zones interconnecting with the central zone by microfluidic channels on the $\mu \mathrm{PAD}$ (Fig. 12A). During the fabrication process, $3 \mu \mathrm{L}$ of 


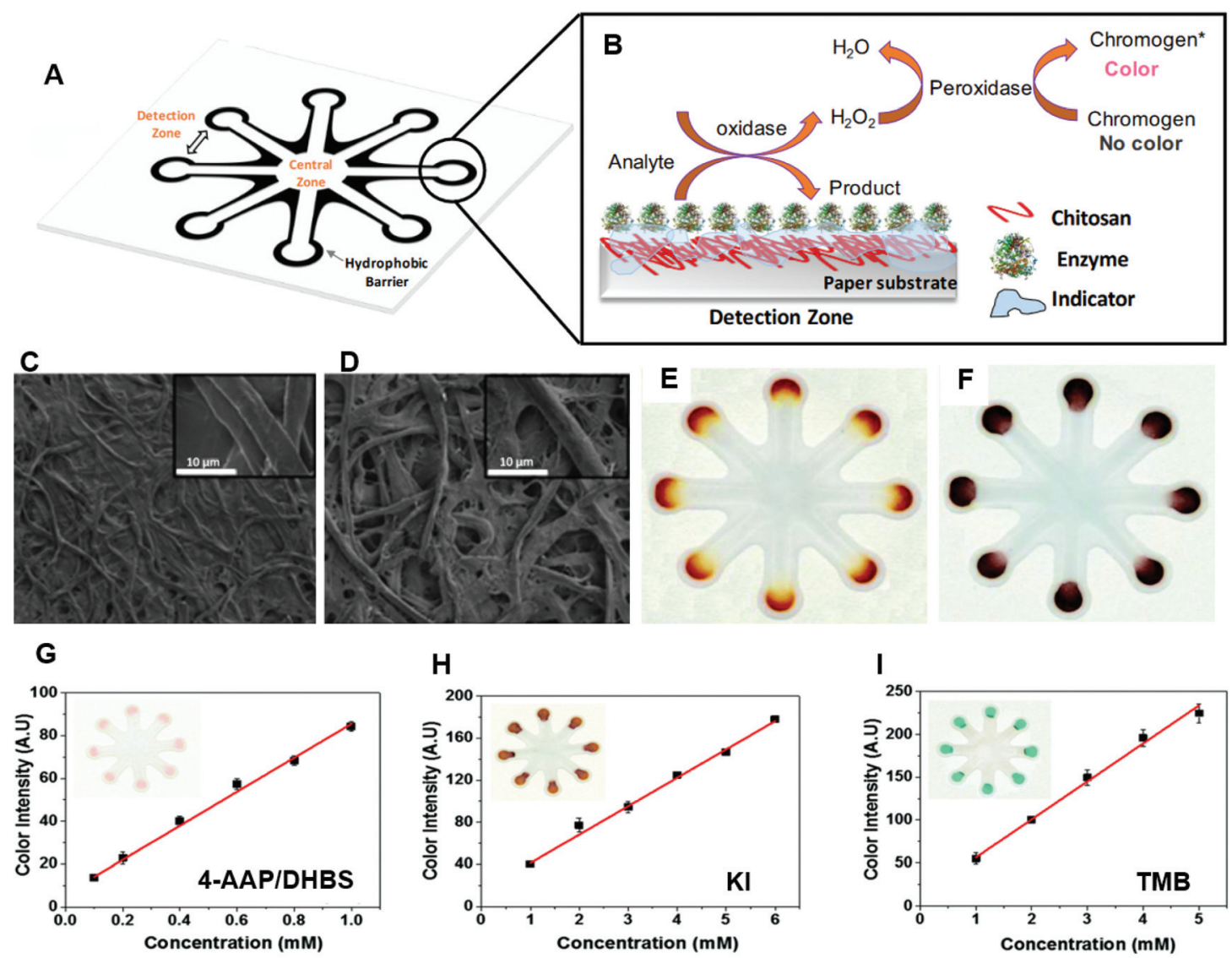

Fig. 12 Experimental data and working principles of the colorimetric sensing of glucose and uric acid on microfluidic paper-based analytical devices $(\mu \mathrm{PAD})$. (A) The schematic configuration of the $\mu$ PAD consists of eight identical detection zones of $5 \mathrm{~mm}$ diameter each, and each connecting with the central zone via microfluidic channels $(3 \mathrm{~mm}$ width and $10 \mathrm{~mm}$ length) in the configuration of $\mu$ PAD. The dimension of the whole $\mu$ PAD was $45 \mathrm{~mm} \times 45 \mathrm{~mm}$. (B) The mechanism of the colorimetric sensing for glucose and uric acid (UA). The morphology of the paper device (C) with and (D) without cellulose were characterized by using a field emission scanning electron microscopy (FESEM) to maintain the surface characterization. Scale bar: $10 \mu \mathrm{m}$. (E) Photograph of the colorimetric glucose-sensing result when chitosan did not cooperate with cellulose; (F) The colorimetric result when glucose sensing with cellulose, wherein the relative standard deviation decreased from $46 \%$ to $11 \%$. The colorimetric results when using different chromogenic solutions including (G) 4-AAP/DHBS, (H) KI, and (I) TMB. All of the indicators provided linear responses with the concentration range from 0.1-1.0 mM (4-AAP/DHBS), 1.0-6.0 mM (KI) and 1.0-5.0 mM (TMB). 4-AAP/DHBS indicated the highest analytical sensitivity $\left(86 \mathrm{AU} \mathrm{Mm}^{-1}\right.$ ) and the lowest limit of detection of $23 \mu \mathrm{M}$. Copyright 2016, Royal Society of Chemistry.

chitosan solution was impregnated on the detection zones to incorporate the colorimetric sensing of glucose and UA (Fig. 12B). The characterization of the paper device, such as morphology (Fig. 12C and D), was studied with the aid of a field emission scanning electron microscopy (FESEM). ${ }^{109}$ With a series of optimizations, the chitosan concentration was determined as $0.5 \%\left(\mathrm{mv}^{-1}\right)$ and the sample volume used was stated to be $c a$. $8.7 \mu \mathrm{L}$ with a set reaction time of $15 \mathrm{~min}$. The signal intensity and color uniformity of the glucose sensor was developed when the chitosan was incorporated with cellulose (Fig. 12E and F). ${ }^{109}$ Moreover, different chromogenic solutions were examined to obtain a better colorimetric result, and 4-AAP/DHBS, KI, and 3,3',5,5'-tetramethylbenzidine (TMB) were employed during detection as colorimetric indicators (Fig. 12G-I). 4-AAP/DHBS was eventually selected as it possessed the highest analytical sensitivity $\left(86 \mathrm{AU} \mathrm{mM}^{-1}\right)$, and the lowest limit of detection of $23 \mu \mathrm{M}$. Finally, the proposed $\mu \mathrm{PAD}$ was utilized for monitoring glucose levels in tear samples of four non-diabetic patients with the indicator (4-AAP/DHBS), which were $190 \pm 10 \mu \mathrm{M}, 380 \pm 10 \mu \mathrm{M}, 250 \pm 10 \mu \mathrm{M}$, and $130 \pm$ $10 \mu \mathrm{M}$. After the evaluation of possible potential interferences (e.g. ascorbic acid, lactate, and uric acid), the outcomes were all stated to agree with normal ranges of glucose found in tears in other works of literature, suggesting that the results are reliable. Therefore, this $\mu \mathrm{PAD}$ can be evaluated as a potential colorimetric sensing technique for tear fluid glucose monitoring in the future. Other $\mu$ PADs were also employed for tear glucose diagnosis, one colorimetric sensor was wax-printed on paper and modified with chitosan in acetic acid using TMB as the chromogenic reagent. ${ }^{111}$ The colorimetric sensor achieved a sensitivity of $84 \mathrm{AU} \mathrm{mM}^{-1}$ within a glucose detection range of 0.1-10 mM, and $50 \mu \mathrm{M}$ of glucose was found to be the detection limit. These paper-based tests and the resulting characteristics of these sensors have demonstrated a possibility for the clinical application of point-of-care devices, in particular for the use of tear fluid-analyzing contact lens sensors. Moreover, 
enzyme-linked immunosorbent assay (ELISA) can also be conducted on paper-based platforms to monitor concentrations of typical biomarkers. ${ }^{112-115}$ If the paper-based devices can be integrated into a contact lens sensor, this could be utilized to detect a wide range of biomarkers (Table 1) in tear fluid. For instance, detection of BDNF in tears was stated to be capable of diagnosing glaucoma, as glaucoma can be regarded a neurodegenerative disease. ${ }^{84,116}$ With the application of a successful paper-based ELISA, BDNF can be tested on a $\mu$ PAD of ELISA, and the assay could be implemented onto a contact lens in order to monitor tear fluid. However, more potential characteristics of $\mu$ PADs need to be explored with the aim of fabricating contact lens sensors for tear monitoring and diagnostics at the POC platform.

3.2.2. Fluorescence sensing. Fluorescence occurs when an excited molecule, atom, or nanostructure absorbs irradiant radiation of a certain wavelength and relaxes to a lower energy state (ground state) by the emission of a photon. There are several common principles that indicate a strongly fluorescent molecule. Firstly, the absorption should take place under a wavelength that is long enough to ensure that dissociation cannot occur, because if the absorption energy of the molecule is greater than the cleavage energy of its least stable bond, then no fluorescence will be detected. Secondly, the rate of radiation must be faster than intramolecular energy transfer. Certain other geometrical factors, such as rigidity and planarity, may also influence the efficiency of fluorescence. ${ }^{117}$ Therefore, in a micro-second fluorescent reaction with transitions from the singlet excited state to the ground state, the wavelengths of excitation and emission photon are highly reliant on the chemical composition and the attribution of the target fluorophore, and fluorescence sensing is highly molecule-specific. Hence, fluorescence-sensing contact lens sensors have been studied and utilized for monitoring various analytes in tears to indicate ocular diseases, such as dry eye disease and diabetic retinopathy.

As the quantitative analysis of analytes in tear fluid is important for early ocular disease diagnosis in the POC platform, typical fluorescent scleral contact lens sensors were constructed to monitor physiological levels of $\mathrm{pH}, \mathrm{Na}^{+}, \mathrm{K}^{+}, \mathrm{Ca}^{2+}, \mathrm{Mg}^{2+}$, and $\mathrm{Zn}^{2+}$ ions. ${ }^{100,151}$ They provided quantitative data on a smartphone readout. ${ }^{100,151}$ One of the advantages of this type of contact lens sensor is its ability to assess the severity stages of dry eye disease and differentiate its subtypes. The contact lens sensors consisted of six concavities carved by laser ablation. Different fluorophores were used with a unique mechanism for the detection of individual concentrations (Fig. 13A-F). Benzene dicarboxylic acid (BDCA) was applied as a fluorescent $\mathrm{pH}$ probe $\left(\mathrm{p} K_{\mathrm{a}} 7.5\right)$, as BDCA can produce a $\mathrm{pH}$-dependent emission shift from yellow-orange $(\lambda=580 \mathrm{~nm})$ to deep $\operatorname{red}(\lambda=640 \mathrm{~nm})$ because of the deprotonation mechanism. As a result, the $\mathrm{pH}$ probe displayed a decrease in fluorescence intensity when the $\mathrm{pH}$ increased from 5.0 to 9.0 with $50 \mu \mathrm{M}$ of BDCA. A sensitivity of $0.12 \mathrm{pH}$ unit was determined within the $\mathrm{pH}$ range of 7.0-8.0. Moreover, the effects of BDCA pH probe on different electrolytes were evaluated, and $\mathrm{Na}^{+}, \mathrm{K}^{+}, \mathrm{Ca}^{2+}, \mathrm{Mg}^{2+}$ and $\mathrm{Zn}^{2+}$ ions were found to show slight change but $\mathrm{Fe}^{3+}, \mathrm{Fe}^{2+}$, and $\mathrm{Cu}^{2+}$ ions exhibited obvious decreases. Finally, the $\mathrm{pH}$ probe $(2 \mu \mathrm{L}$, $50 \mu \mathrm{M}$ ) was functionalized in the concave of the contact lens. A similar fabrication and testing process was conducted for the targeted ions (Fig. 13B-F). As a result, the crown ether derivatives were used for selecting $15.6 \mathrm{mM}$ of $\mathrm{Na}^{+}$with a detection range of $0-100 \mathrm{mM}$, and also for $\mathrm{K}^{+}$detection in the range of 0-50 mM with a selectivity of $8.1 \mathrm{mM}$. A 1,2 bis(o-amino phenoxy) ethane- $N, N,-N^{\prime}, N^{\prime}$-tetraacetic acid-based probe was utilized for $\mathrm{Ca}^{2+}$ detection within a detection range of 0.50-1.25 $\mathrm{mM}$ and 0.02-0.05 $\mathrm{mM}$ in sensitivity, and a 5-oxazolecarboxylic acid probe was produced for sensing $\mathrm{Mg}^{2+}$ ion with an obtained sensitivity of $0.10-0.44 \mathrm{mM}$ ranging between 0.5 and $0.8 \mathrm{mM}$. Additionally, the $N$-(2 methoxyphenyl)iminodiacetate $\mathrm{Zn}^{2+}$ ion sensor showed a sensitivity of $1 \mu \mathrm{M}$, and the range of detection was $10-20 \mu \mathrm{M} .{ }^{100}$ Another exciting advantage of this research is the development of the portable readout device. During the experiment, the research group fabricated a handheld ophthalmic readout device consisting of light-emitting diodes (LEDs) and bandpass filters to excite and read the sensor. Moreover, a smartphone camera was coupled with the use of the constructed readout device to deliver quantitative measurements. The scleral contact lens sensor was then carried in the artificial tear fluid for the diagnosis and monitoring of dry eye disease, in particular to assess severity stages and disease subtypes, i.e. meibomian gland dysfunction (MGD) or lacrimal gland dysfunction (LGD). It is advantageous that the fluorescent data can be converted to a readable outcome on smartphones, allowing patients to receive data related to their ocular conditions at any time required.

Other electrolytes such as chloride ions were studied by other research groups. One of these groups built a silicone hydrogel (SiHG) contact lens sensor to monitor $\mathrm{pH}$ and $\mathrm{Cl}^{-}$ ion levels. During the fabrication and evaluation process, three sensing probes were detected for characterizing the SiHG sensor. 1-Anilinonaphthalene-8-sulfonic acid (1,8-ANS, ANS) and a 4-(1-octylamine)-7-nitrobenzoxadiazole (NBD-C18) were applied for polarity sensitive probes. The hydrophobic $\mathrm{pH}$ sensing probe, 6HQ-C18, was compared to the hydrophilic 6HQ-C3 probe used as a control. A 6-methoxyquinolinium-containing (SPQ) probe was utilized for chloride ion sensing. ${ }^{118}$ Both the $\mathrm{pH}$ and the $\mathrm{Cl}^{-}$ion sensing mechanisms used the C18 and C3 alkyl chain for hydrophobic and water-soluble fluorescence detection respectively. Instead of connecting the ions with a soft hydrogel contact lens, one type of SiHG was selected for having a higher $D_{\mathrm{k}}$ value and silicone content (52\%), which was considered beneficial for long-term continuous wear. In addition, both polar and non-polar interface regions have existed in SiHG, whereas no such region is present in a hydrogel contact lens. ${ }^{118}$ As a result, the hydrophobic ion-sensitive fluorophores can bind strongly to SiHG lenses and aqueous solutions could not wash out these fluorophores. However, the sensitivity and limit detection range were not reported in experimental data. The same group recently carried out research into detecting both $\mathrm{Na}^{+}$and $\mathrm{Cl}^{-}$ions within the SiHG lenses. The fabrication process and detection 
A

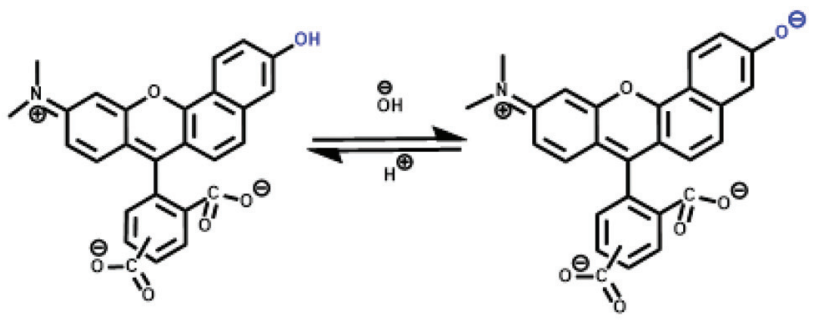

B<smiles>[R]N1CCOCCOCCN([R])CCN(P)CCOCCN(P)CCOCCOCC1</smiles>

D<smiles>[R]c1ccc(N(C(=O)[O-])C(=O)[O-])c(OCC(=O)N(CC(=O)[O-])c2ccc([N+](=O)[O-])cc2OCC(=O)[O-])c1</smiles><smiles>[R9]OC(=O)C(Cl)=CC</smiles><smiles>COc1ccc(OC)c(NC(=O)c2ccc(-c3c4cc(Cl)c(=O)cc-4oc4cc([O-])c(C)cc34)c(C(=O)O)c2)c1</smiles>

C

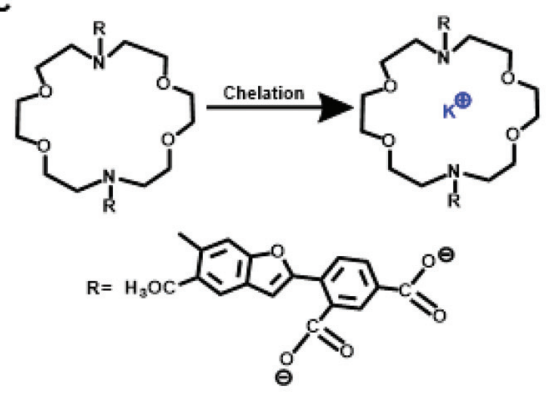

E<smiles>[Y]OC(=O)COc1cc([R])ccc1N(CC(=O)O[R])CC(=O)O[Y]</smiles><smiles>[R6]Oc1cc2oc3cc(=O)c(F)cc-3c(C)c2cc1[OH2+]</smiles>

F<smiles>O=C([O-])c1cnc(C2=CCCO2)o1</smiles>

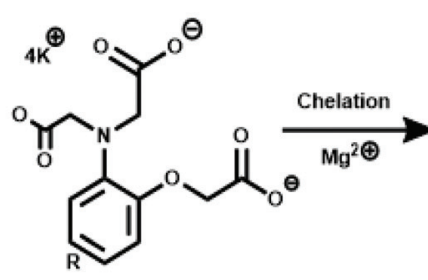<smiles>O=C([O-])COc1ccccc1N(CC(=O)[O-])CC(=O)[O-]</smiles>

G

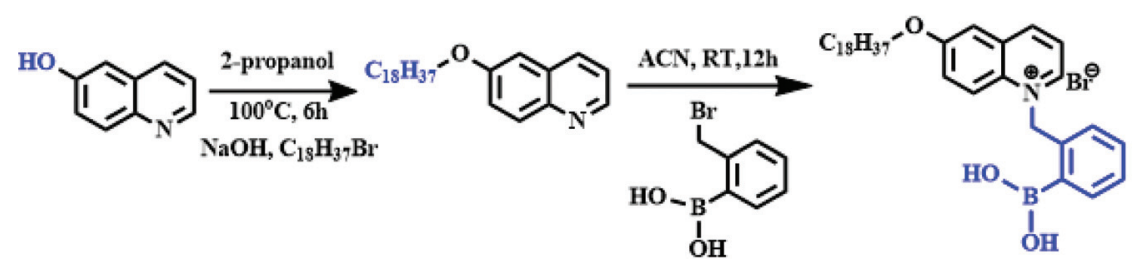

Fig. 13 A summary of fluorescent sensing mechanisms and fluorophore synthesis process. (A) pH sensing (B) $\mathrm{Na}^{+}$sensing (C) $\mathrm{K}^{+}$sensing (D) $\mathrm{Ca}{ }^{2+}$ sensing (E) $\mathrm{Zn}^{2+}$ sensing (F) $\mathrm{Mg}^{2+}$ sensing (G) forming mechanism for fluorophore Quin-C18.

methods were similar, but the binding fluorophores were adjusted, where the applied chloride-sensitive fluorophore contained an octadecyl (OD) side chain and was hydrophobically binding to the non-polar region of the SiHG lenses. ${ }^{119} \mathrm{~A}$ covalent conjugation was formed between sodium green (SG) and poly-L-lysine (PL) to monitor sodium-ion-sensitive fluorophores. A wider detection ranges of both $\mathrm{Na}^{+}$and $\mathrm{Cl}^{-}$ion sensors were reported, and the most important achievement 
was the independent response from $\mathrm{Na}^{+}$and $\mathrm{Cl}^{-}$ion detection on a single lens. These series of research provided a comprehensive idea about the interfacial region of silicone hydrogel contact lenses, which could be applied to monitor other analytes within tear fluid for ocular disease diagnosis.

Other fluorescent contact lens sensors have been proposed, including glucose sensing in tear fluid. For instance, a glucose-sensitive silicone hydrogel contact lens was recently established for continuous tear fluid glucose-monitoring glucose in the near term. Compared to the electrochemicalbased sensor module, ${ }^{104}$ this research simplified the monitoring mechanism and used the glucose-sensitive fluorophore (Glu-SFs), Quin-C18, inserted into SiHG lenses. ${ }^{120}$ During the fabrication process, the interpenetrating polymer network (IPN) that existed in SiHG lenses was evaluated with almost pure silicone and water regions using a polarity-sensitive probe, Prodan. The glucose-sensitive fluorophore Quin-C18 (Fig. 13G) was then synthesized and consisted of a hydrophobic chain to localize the probe at the interfacial region. ${ }^{120}$ This glucose contact lens sensor can be applied for the measurement of varying glucose concentrations in an in vitro platform. Significantly, the utilized fluorophore (Quin-C18) bound strongly to lenses with a negligible leaching rate even after several rinses. Furthermore, the measurements of glucose sensing were similar even after lenses underwent three months of storage in water. Therefore, this is likely to have beneficial applications for tear glucose-sensing in POC settings in the near future. Therefore, fluorescence sensing via contact lens sensors, and the ability to obtain the data via handheld readers, provides the potential to explore the physiological properties of human eyes and further understand the pathophysiology of ocular diseases, as well having substantial potential clinical applications.

Instead of applying the fluorescent sensing technologies ono contact lenses, microfluidic paper-based device is also one of the typical applications that have been studied for tear diagnosis. Lactoferrin detection without antibody is one of the most significant study for fluorescent $\mu$ PADs. The microfluidic patterns on $\mu$ PADs were fabricated with the inkjet printer and a UV-curable ink. ${ }^{121}$ The fabricated paper-based sensor can provide a limit of detection of lactoferrin at $0.3 \mathrm{mg} \mathrm{mL}$ within a detection range from 0.5 to $3 \mathrm{mg} \mathrm{mL} \mathrm{m}^{-1}$. Then, a distance-based lactoferrin was also developed form a similar method. An even lower limit of detection of lactoferrin was achieved at $0.1 \mathrm{mg} \mathrm{mL} \mathrm{m}^{-1}$ using this kind of paper-based sensing technology, and the detection range was $0-4 \mathrm{mg} \mathrm{mL}^{-1}$ of lactoferrin. ${ }^{122}$ With the achievement of detection for tears using $\mu$ PADs, the further development could be then explored for implementation of paper-based sensors onto contact lenses to achieve the real-time point-of-care diagnostic platform for ocular diseases.

3.2.3. Electrochemical sensing. Microsystem-based contact lens sensing techniques have been well-established in the semiconductor industry recently. Due to their advantages, including reduced waste production, miniaturized analytes requirements, fast response times, and low-cost manufacture, electrochemical sensing techniques are increasingly popular for use in tear monitoring in POC platforms. Electrochemical sensors use electrodes as the transducer elements, and can also be applied in combination with other techniques such as nanotechnology, to increase precision, selectivity, specificity, and sensitivity. These sensors can also be employed for quantifying glucose concentrations in tear fluid with the aid of 3-electrode systems. Glucose oxidase (GOD) a common enzyme used for glucose electrochemical sensing, and the electrical charge can be created as the enzyme-substrate complex forms. Three main reactions are included in glucose electrochemical sensing (eqn (3)-(5)), and the magnitude created by the charge is proportional to the concentration of oxidized glucose.

$$
\begin{gathered}
\text { D-glucose }+\mathrm{O}_{2} \stackrel{\text { GOD }}{\longrightarrow} \mathrm{H}_{2} \mathrm{O}_{2}+\text { D-gluconolactone } \\
\text { D-gluconolactone }+\mathrm{H}_{2} \mathrm{O} \rightarrow \text { D-gluconic acid } \\
\mathrm{H}_{2} \mathrm{O}_{2} \rightarrow 2 \mathrm{H}^{+}+\mathrm{O}_{2}+2 \mathrm{e}^{-}
\end{gathered}
$$

An electrochemical contact lens sensor monitoring the concentration of glucose in artificial tear fluid was reported. ${ }^{123}$ During the fabrication process, the typical photoresist and thin metal were deposited onto a poly (ethylene terephthalate) (PET) film to form the circuitry under heat moulding in contact lens shape (Fig. 14A). The finished contact lens sensor comprised sensing structure, interconnects, and electrode pads, which include reference electrode (RE), working electrode (WE), and counter electrode (CE). Titania sol-gel was utilized for immobilizing GOD and was covered by a Nafion layer. The wireless readout was developed from a computer chip and obtained the power from far-field electromagnetic radiation by a wireless device that supplied energy $(3 \mu \mathrm{W})$ to the sensor at $15 \mathrm{~cm}$. After multiple tests, the sensor performed at a high sensitivity of $240 \mu \mathrm{A} \mathrm{cm} \mathrm{cm}^{-2} \mathrm{mM}^{-1}$ with a response time of $20 \mathrm{~s}$. Within a glucose concentration range of $0.1-0.6 \mathrm{mM}$ in the tear film, the sensor had a sensitivity of $0.01 \mathrm{mM}$ and provided an acceptable accuracy under the presence of interference including urea, ascorbic acid, and lactate with a relatively high concentration of glucose $(>0.3 \mathrm{mM})$. Moreover, a dual glucose electrochemical sensor (GOD/DeGOD) was then formed with a similar structure and process as in Fig. 14, and the detection range of glucose was claimed as $0-2 \mathrm{mM}$ with an implemented low-power $(3 \mu \mathrm{W})$ sensor read-out device. ${ }^{124}$

Similarly, another type of electrochemical contact lens sensor for in situ monitoring of tear contents was established and evaluated in vivo with rabbits. ${ }^{125}$ The sensor consisted of an enzyme (GOD) immobilized electrode binding with a polydimethylsiloxane (PDMS) layer (Fig. 14B and C). A copolymer mixture consisting of 2-methacryloyloxyethyl phosphorylcholine (MPC) and 2-ethyl hexyl methacrylate (EHMA) was prepared for coating and immobilizing the sensing material (GOD). The combination of flexible biosensor and the soft contact lens sensor made a complete structure of this kind of electrochemical sensor. According to the results, the output current had high correlation with a glucose concentration range of $0.03-5.0 \mathrm{mM}$. Moreover, it was possible for the sensor 
A

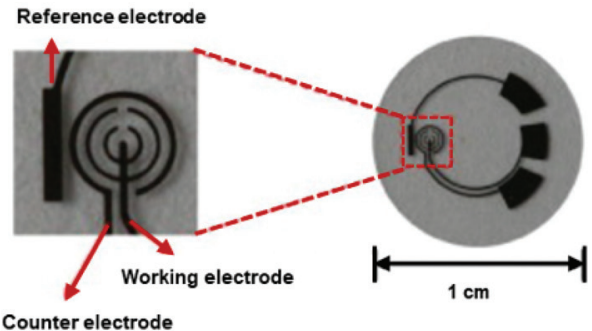

Counter electrode

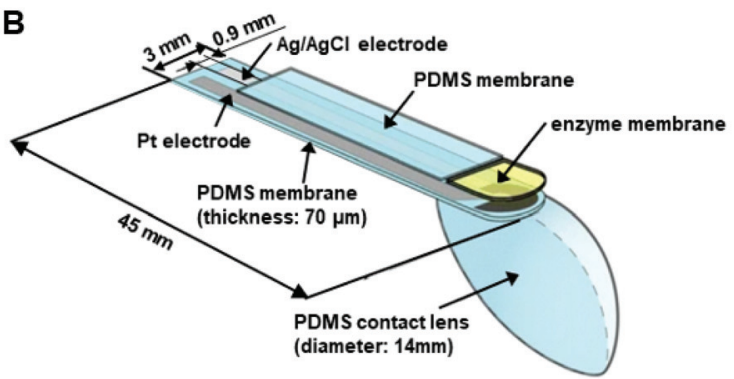

B

D

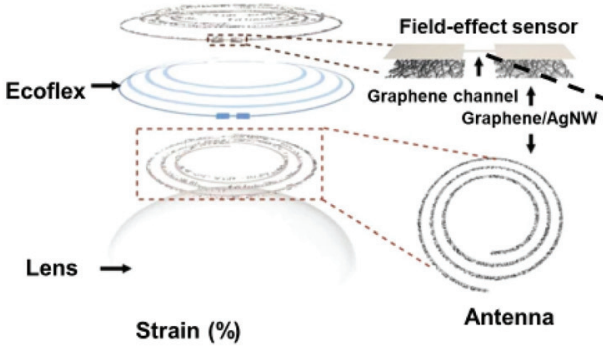

C

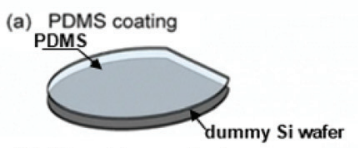

(b) Pt and Ag sputtering
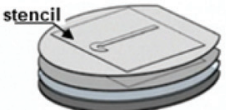

c) Insulation and chloride treatment

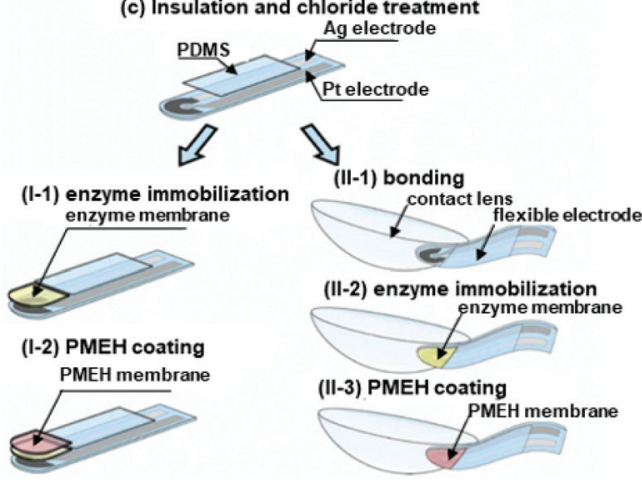

Fig. 14 Electrochemical sensing fabrication technologies. (A) The glucose and L-lactate contact lens sensor comprising the sensing structure, interconnections, and electrode pads: reference electrode (RE), working electrode (WE), and counter electrode (CE). Copyright 2012, Elsevier. (B) The structure and constituents of the PDMS contact lens sensor for tear glucose monitoring. (C) The schema of the fabrication process of the PDMS contact lens sensor. Copyright 2011, Elsevier. (D) The scheme of the multifunctional contact lens sensor was used for glucose and intraocular pressure monitoring. The mechanism for glucose sensing was the same as in its previous description in this review. Copyright 2017, Nature Communications.

to detect a change in tear glucose levels with 10 min delay and peaked after $55 \min ^{126}$ With an in vivo rabbit ocular monitoring process, the sensor behaved well in both static state and dynamic state, and the tear turnover was calculated as $29.6 \pm$ $8.42 \% \mathrm{~min}^{-1}$ after an estimation of the glucose tear level $(0.12 \mathrm{mM})$ in rabbit models.

More recently, a multifunctional contact lens sensor was developed for continuous glucose and IOP sensing in rabbit tears to evaluate the functions in vivo and in vitro. ${ }^{127}$ Instead of linking lens-shaped polyethylene terephthalate or PDMS substrates, the contact lens sensor was graphene-based and cooperating with silver nanowires (AgNWs). The glucose monitor technique was based on GOD-conjugated field-effect transistor sensing, and a high response was obtained within the concentration range of $0.1-0.6 \mathrm{mM}$ under a sensitivity of $1 \mu \mathrm{M}$. As the fabricated sensor for glucose monitoring comprised the resistance (R), inductance (L), and capacitance (C) circuit, the IOP was monitored by insertion of a silicone elastomer (Eco flex) layer between the two inductive spirals forming a sandwich structure to detect the structural change on the eye (Fig. 14D).
Also stated was the possibility of integrating the pyrenebinding biomarker receivers onto graphene in order to detect more analytes in tears, with potential applications in the future evolution of multifunctional contact lens sensors for ocular diagnosis. The achievement of in vivo examination of this type of sensor also provided the evidence of biocompatibility for clinical applications. The wearable contact lenses are advantageous in the permeability of higher oxygen and water compared to PDMS substrates or polyethylene terephthalate in lens shapes. And the ultra-thin parylene with around $500 \mathrm{~nm}$ thickness, was selected as a layer material of graphene channel due to its superiority in mechanism (strength and stretchability) and high intraocular biocompatibility. Besides, one of the hormone kinds of biomarker, cortisol, was also detected using graphene field-effect transistor. ${ }^{128}$ The fabricated electrochemical sensor was then developed further for IOP quantitively demonstration on glaucoma and treatments of diabetes diagnosis and monitor. ${ }^{129,130}$ As one of the new treatments for diabetes was indicated as intraocular islet transplantation, one smart wireless contact lens sensor was then 
fabricated for real-time monitoring of the IOP before, during and after the transplantation and the fabricated sensor was then implemented in live rats. ${ }^{130}$ Moreover, a strain sensor was implemented onto the rigid region of load coil of the contact lens and plus the inductive coil to form the reading loop. Instead of constructing a sandwich structure of IOP and glucose contact lens sensor, a development of transparent IOP sensing type of contact lens was also indicated and was examined clinically with ten patients. ${ }^{129}$ The accuracy of the results was compared with a commercially available tonometer and the intraclass correlation coefficient was indicated as 0.888 . Further investigation and therapeutic development of other ocular diseases on wireless contact lens sensor, such as chronic ocular surface inflammation, was also reported. The fabricated sensor can not only real-time monitoring the variation of concentration of matrix metalloproteinase-9 (MMP 9), but also implemented the heat patch for release the hyperthermia treatments automatically. ${ }^{131}$ All readout data of smart contact lens sensors were coordinated with live animals and human patients which provided the feasible and reliable biocompatibility of the research. The achievement of therapeutic level of sensor was remarkable for electrochemical sensing in ophthalmology.

Biosensors for lactate monitoring in tears have been studied and the sensing mechanism is almost the same as glucose sensing (GOD), as lactate oxidase (LOx) can be used to convert L-lactate and oxygen into pyruvate and hydrogen peroxide. In addition, with a sufficient potential difference, the produced hydrogen peroxide can be oxidized to form oxygen and hydrogen ions at a platinum electrode. The PET contact lens L-lactate sensor was lithographically patterned by a positive resist (AZ4620) to form the circuit. ${ }^{106}$ The LOx was fixed in the platinum sensing area by covalent bonds between the enzyme and bovine serum albumin (BSA) using glutaraldehyde (GTA), and the sensor was coated with medical-grade polyurethane. The sensor exhibited a fast response time of $35 \mathrm{~s}$ and an average sensitivity at around $53 \mu \mathrm{A} \mathrm{mM} \mathrm{m}^{-1} \mathrm{~cm}^{-2}$ within a tear lactate level under physiological conditions. To prevent the interference of ascorbic acid, a dual-sensor was produced with $\mathrm{Ti} / \mathrm{Pd} / \mathrm{Pt}$ electrode material and functionalized with active enzyme (3 $\mu \mathrm{L}$ phosphate-buffered saline (PBS) with $2 \%$ LOx, $3 \% \mathrm{BSA}$, and $0.3 \%$ GTA) to recognize both L-lactate and ascorbic acid. The second sensor was treated with PBS, BSA, and GTA to detect ascorbic acid as a control group, so it could be compensated for the interference signal. With a functional evaluation of physiological ocular temperature, the response of the sensor was reported as stable for up to $24 \mathrm{~h}$.

Nanoparticles can also be employed in contact lens sensors to monitor analytes in tear fluids. A nanoparticle-embedded contact lens sensor that utilized GOD and cerium oxide(III) was proposed for tear glucose measurement with the aid of spectroscopy. ${ }^{132}$ The sensor detected under $0.0 \mathrm{mM}, 0.6 \mathrm{mM}$, $1.2 \mathrm{mM}$, and $2.4 \mathrm{mM}$ of glucose mixed in $5 \mu \mathrm{L}$ of artificial tear fluid, and presented positive results compared to the experimental data between diabetic and non-diabetic mouse groups.
Due to a complex fluid environment and other interference influences in tears such as evaporation, atmospheric humidity, and 'reflex' or 'emotional' tears, some simplified modules of a sensor may not be able to provide precise concentration levels of analytes in the eyes. In order to get more informative data about the introduction, mixing, and drainage of the target analytes in lacrimal fluid using an electrochemical sensing method, a real-speed spatiotemporal electrochemical contact lens sensor was developed for ocular diagnosis. ${ }^{133}$ The sensor consisted of four microelectrode arrays in the four quadrants on the soft contact lens (Fig. 15). Each individual microelectrode was formed by 33 gold micro-discs of $30 \mu \mathrm{m}$ diameter and connected to a gold counter electrode (Fig. 15A-D). Various ferrocene-methanol $(\mathrm{FeMeOH})$ solutions were mixed with $0.1 \mathrm{M}$ potassium chloride ( $\mathrm{KCl}$ ) in deionized water, and $0.5 \mathrm{mM}$ of $\mathrm{FeMeOH}$ were utilized to check the electrochemical behavior on each bare electrode separately using a standard cyclic voltammetry $\left(0 \mathrm{~V}-5 \mathrm{~V}-0 \mathrm{~V}\right.$, at $\left.0.1 \mathrm{~V} \mathrm{~s}^{-1}\right)$. Within a concentration range of $10-500 \mu \mathrm{M}$ of $\mathrm{FeMeOH}$, a linear current was reported, where $0.1 \mathrm{M}$ of $\mathrm{KCl}$ was measured as 'blank'. The spatiotemporal sensing was discussed on the results obtained from a beaker (Fig. 15E), and a total rinse was completed within $10 \mathrm{~s}$. In the final step, a modeled eye experiment was conducted and reported a slight elevation in concentration from the model (Fig. 15F). However, this experiment offered a method for checking the variation of analytes in tear fluid dynamically and with more accurate data for analysis. More analytes and biomarkers could be implemented for ocular diagnosis.

It is worth mentioning that instead of real biomarkers detection and IOP evaluation, electroretinogram examination could also be achieved using the commercial soft contact lens. One smart contact lens sensor was fabricated and developed as a non-invasive all-printed stretchable corneal contact lens senor. ${ }^{134}$ The seamlessly electrochemical anchoring mechanism was introduced to a soft contact lens ensuring a highfidelity records on the signals of full-field electroretinogram within human eyes. The superiority of this device was stated as non-invasive compared to the modern rigid sensor, as the topical anesthesia or a speculum would be required for original sensor. Moreover, the signal quality and conformability were outstanding in compare with the clinical standard. This type of soft contact lens sensor would provide one of the future studies directions in ophthalmology, especially in ocular electro-diagnose platform.

3.2.4. Photonic-based sensing. Photonic crystals (PCs) are highly ordered materials and have a periodically modulated dielectric constant. These nanostructures have photonic-band gaps that can confine and control the propagation of light, hence photonic crystals have been considered as a potential research area for future study. Based on the differences in the refractive index and period in space, photonic crystals are divided into three groups which are one-dimensional (1D), two-dimensional (2D) and three-dimensional (3D). Generally, various layers are deposited or stuck together to form 1D photonic crystals, 2D photonic crystals are formed from photo- 
A

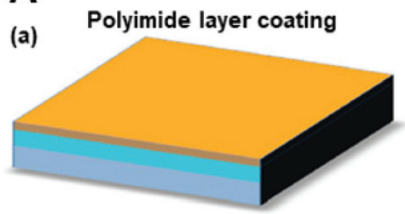

(b) Metal layer coating

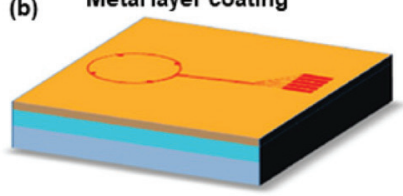

(c) Wet etching and stripping

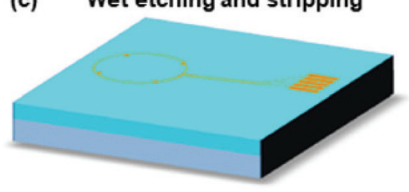

B

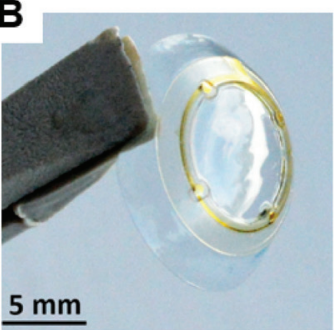

(d)

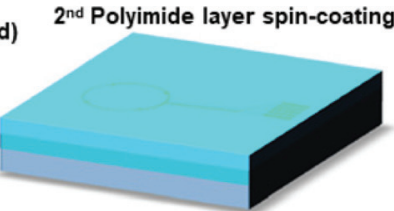

(e) On-put photoresist

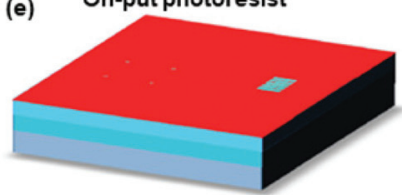

(f)

Shaped by picosecond laser

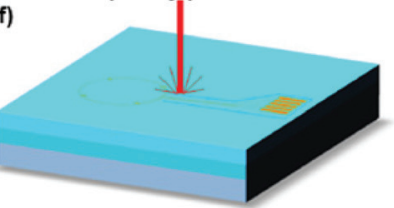

C

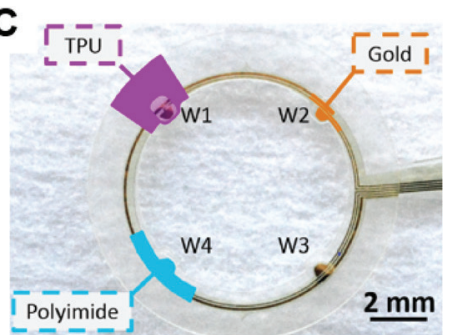

(g)

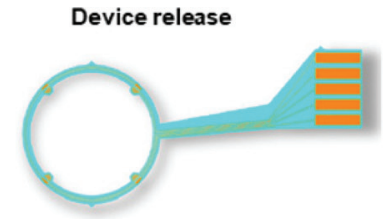

(h)

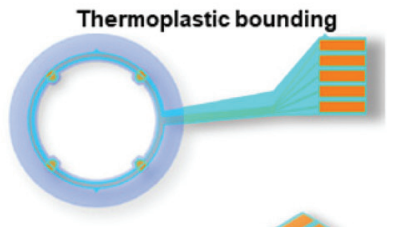

Device thermoformed

(i)
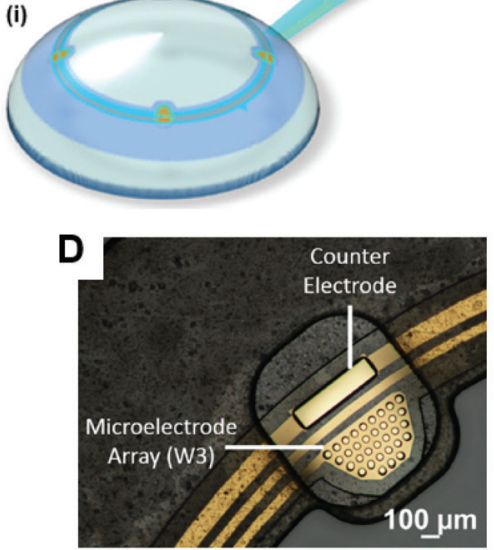

E Beaker Experiment

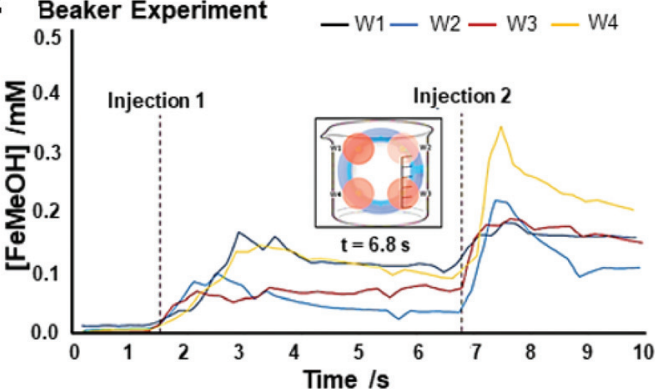

F

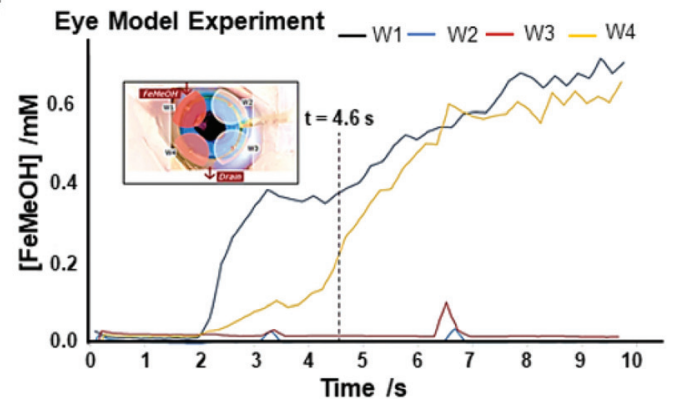

Fig. 15 Fabrication mechanism schemes and the experimental data of the spatiotemporal electrochemical contact lens sensor (ESCL). (A) The fabrication process and analytical data of the spatiotemporal electrochemical contact lens sensor. (a) Polyimide (5.5 $\mu \mathrm{m})$ and TiW/Au (50 nm, adhesion layer; $1100 \mathrm{~nm}$ ) layers on a glass wafer; (b) Photolithographic (S1818 resist) patterned to define metal layers; (c) metal layers defined by wet etching and stripping of photoresist; (d) polyimide layer spin-coated over metal layers; (e) thick photoresist (AZ4562, target thickness $10 \mu \mathrm{m}$ ) patterned to define contact pads and electrode sites; ( $f$ ) after reactive ion etch (45 min, $22 \mathrm{sccm} \mathrm{O}, 5 \mathrm{sccm} \mathrm{N}, 350 \mathrm{~m}$ Torr, $75 \mathrm{~W}$ ) and stripping of photoresist, a picosecond laser ( $355 \mathrm{~nm}, 12 \mathrm{ps}, 50 \mathrm{kHz}, 320 \mathrm{~mW}$ ) was used to cut out the device; (g) device released from glass wafer; (h) pre-patterned thermoplastic polyurethane bonded on either side of the device, and device cut to shape using a picosecond laser (355 nm, $12 \mathrm{ps}, 50 \mathrm{kHz}, 400 \mathrm{~mW}, 10$ passes at $40 \mathrm{~mm} \mathrm{~s}^{-1}$ ); (i) device thermoformed to a spherical cap shape and added to hydrogel contact lens. (B) The photograph of the ESCL. (C) The photograph of the unmoulded ESCL consists of four electrodes in different regions. Working electrodes 1-4 (W1-4) indicate different characterisations. (D) The micro-sized photograph of detailed structures on working electrode 3 (W3). (E) Examination of the flow property of the fabricated sensor within a beaker. The calibrated concentration data were obtained for individual working electrodes within $10 \mathrm{~s}$. There were two injections of $\mathrm{FeMeOH}$ into $\mathrm{KCl}$ with initial volume. A red colour shading was presented as the concentration varied. (F) A simulation flow was conducted on an eye model, the $\mathrm{FeMeOH}$ was implemented after an injection of initial $\mathrm{KCl}$. Copyright 2020, Elsevier.

lithography or drilling holes within a suitable substrate, and a $3 \mathrm{D}$ photonic crystal can be fabricated under different methods, for example direct laser writing and different angles of drilling. In the near-decade, photonic crystal-based sensing has been applied for tear monitoring and diagnosis. There are various contact lens sensors constructed in cooperation with photonic structures, such as holographic sensing and crystal arrays.

One-dimensional PCs: holographic gratings. When a white light exhibits an illumination on the PCs, the light will be 
diffracted because of Bragg's Law (eqn (6)) where m indicates the diffraction order, $\lambda$ is the wavelength of the incident light, $n$ is the refractive index of the matrix, $d$ is the spacing of the PC plane and $\theta$ is the received angle of the photodetector.

$$
m \lambda=2 n d \sin \theta
$$

Because the spacing or refractive index changes during diffraction, the light colour will vary as its wavelength shifts. Hence holographic gratings are always used to express minute changes on the PC plane. Such holographic methods have been utilized with photonic structures to quantify the analytes in tears with colour changes for ocular diagnosis. One glucose sensing-based optical contact lens sensor was introduced for continuous monitoring in a point-of-care platform (Fig. 16). The fabrication process was simplified and allowed smartphone readouts. ${ }^{101}$ During the fabrication process, a glucoseselective hydrogel film was facile stamped with a $6 \mu \mathrm{m}$ periodical photonic microstructure and was functionalized with phenylboronic acid. In this way, the volume of the hydrogel varied reflecting the concentration change of glucose, which modulated the periodic constant of the microstructure. According to Bragg's Law, the ultimate change in diffraction adjusted the space between zero- and first-order spot. The individual sensor displayed a sensitivity of $12 \mathrm{~nm} \mathrm{mM}^{-1}$ within a detection range of $0-50 \mathrm{mM}$, and the response time under a saturated state was less than $30 \mathrm{~min}$. After the sensor was integrated onto a commercial contact lens, a short response time of $3 \mathrm{~s}$ was reported within a saturation period of 4 min under a smartphone camera measurement of the reflected power for first-order light diffraction. However, glaucoma can be a disturbance of this type of contact lens sensor in practical use, due to the variation of IOP might affect the periodic structure of the photonic-based sensor.

Multi-dimensional PCs. 2D and 3D photonic structures have also been studied for biosensing technologies. Photonic crystal array sensors are 3D PCs widely explored for diagnosis via tears. These PCs comprise nano-sized particles immobilized within polymer matrices and are formed from crystalline nanospheres which are stacked in order, for example polystyrene and silica. Colloidal crystal arrays are an example of
A

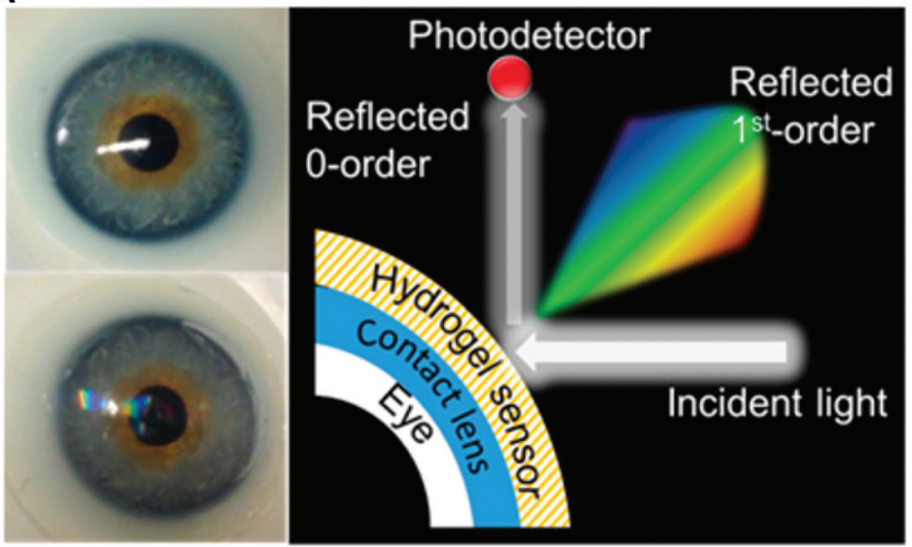

C

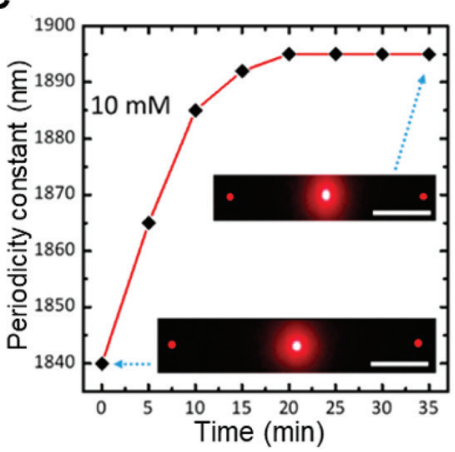

D

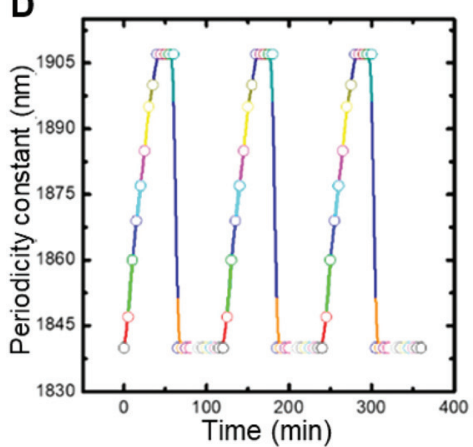

B

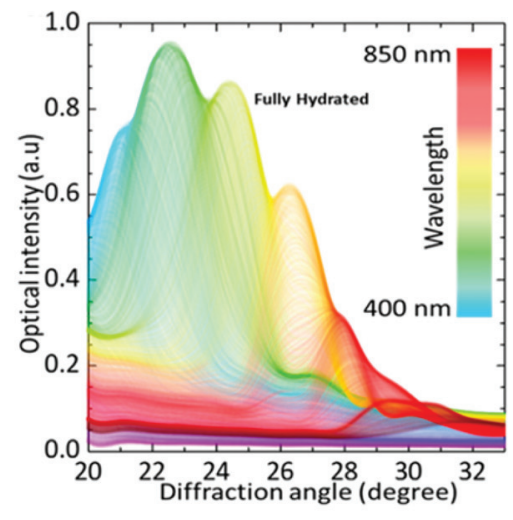

E

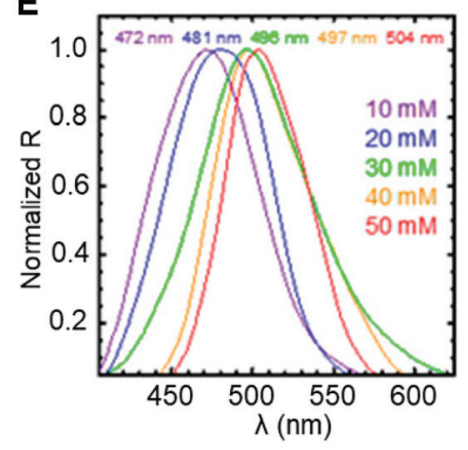

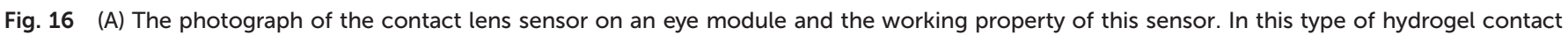

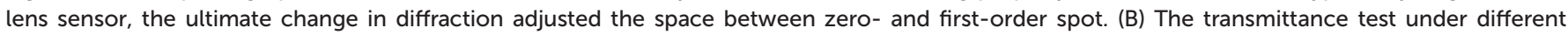

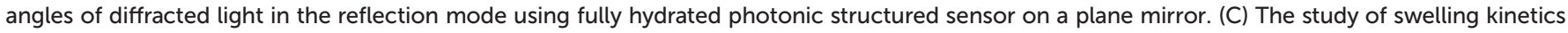

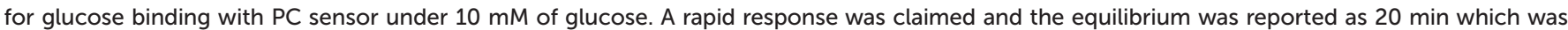

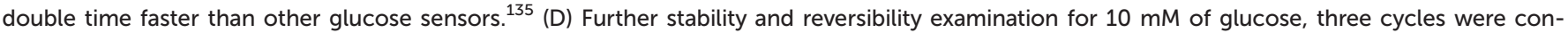

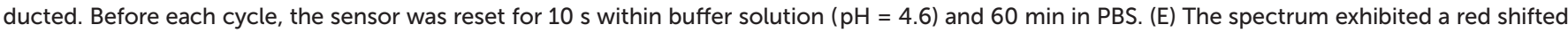
at a certain angle of detection with increasing concentrations of glucose. Copyright 2018, American Chemical Society. 
this and can be fabricated from the evaporation of colloidal solutions by self-assembling the monodispersed particles. For example, a crystalline colloidal array (CCA) embedded in hydrogel matrix sensor and connecting onto a rigid gas permeable (RGP) contact lens was developed for glucose-responsive sensing (Fig. 17A). ${ }^{103}$ The biocompatibility was tested first to check the cytotoxicity of fabricated contact lens sensor to human corneal epithelial cells (HCECs), and the CCK-8 assay was used to collect data quantitatively under different concentrations of GCCA-lens that are cultured from different time (Fig. 17B). Within a detection range of $0-50 \mathrm{~mm}$ of glucose in buffered solution (Fig. 17C), the sensor could diffract light selectively, and had a wavelength shift between $567 \mathrm{~nm}$ and $468 \mathrm{~nm}$ corresponding to a visible colour change in reddish yellow, green, and blue. Amongst these shifts, the visible blue gave a wavelength shift from $525 \mathrm{~nm}$ to $468 \mathrm{~nm}$ within 0-3 mM of glucose (Fig. 17D). A change from green to yellow was highly visible under a $50 \mathrm{~mm}$ glucose concentration. The detection limit for glucose sensing was reported as $0.05 \mathrm{~mm}$. Based on the physiologically-relevant concentration range of glucose in tears (0.1-0.6 $\mathrm{mM})$, the CCA sensor exhibited a good response, wherein a $10 \mathrm{~nm}$ diffraction wavelength shifted from $0 \mathrm{mM}$ to $0.6 \mathrm{~mm}$ in the simulated tear fluid (Fig. 17E). ${ }^{103}$ Although the sensitivity data obtained from tear fluid was onethird lower than that from buffered solution, the sensor can selectively bind to glucose in the presence of other analytes in simulated tear fluid, and future improvements may increase the possibility for practical use.
With the aim of achieving instrument-free detection and monitoring of signs of ocular disease on the POC platform, rather than using biomarker-binding, complex electronics or chemical reactions within tears, a novel structurally coloured contact lens sensor was fabricated. This structurally coloured contact lens sensor displays tunable colours under the broadband light that can directly demonstrate changes in moisture and IOP, which are important criteria in the diagnosis of xerophthalmia and glaucoma respectively. ${ }^{136}$ One of the most important characteristics of this coloured contact lens sensor is its remarkable biosafety and comfort for wearers, as it is constructed from a biocompatible hydrogel (poly(2-hydroxyethyl methacrylate) (PHEMA) without additional chemical pigments. Structural colour, commonly found in nature, arises when unique $3 \mathrm{D}$ periodic structures carry out the wavelengthselective diffraction of light. ${ }^{137-141}$ Both inside and outside moulds were used to prepare the contact lens sensor. During the fabrication process, the monodispersed silica colloidal particles were synthesized in three different sizes: $356 \pm 18 \mathrm{~nm}$, $240 \pm 11 \mathrm{~nm}$, and $180 \pm 9 \mathrm{~nm}$. Then the particles were centrifuged with deionized water and ethanol separately to obtain pure silica colloidal particle suspensions. The different sized monodispersed silica colloidal particle suspensions were subsequently dropped into the outside mould, assembling various colloidal crystal templates, with a complete drying process at $85^{\circ} \mathrm{C}$ in an oven. As a result of the coffee-ring effect, a ringlike colloidal crystal template was produced on the bottom of the mould, ${ }^{142}$ and some of the deposited particles in the
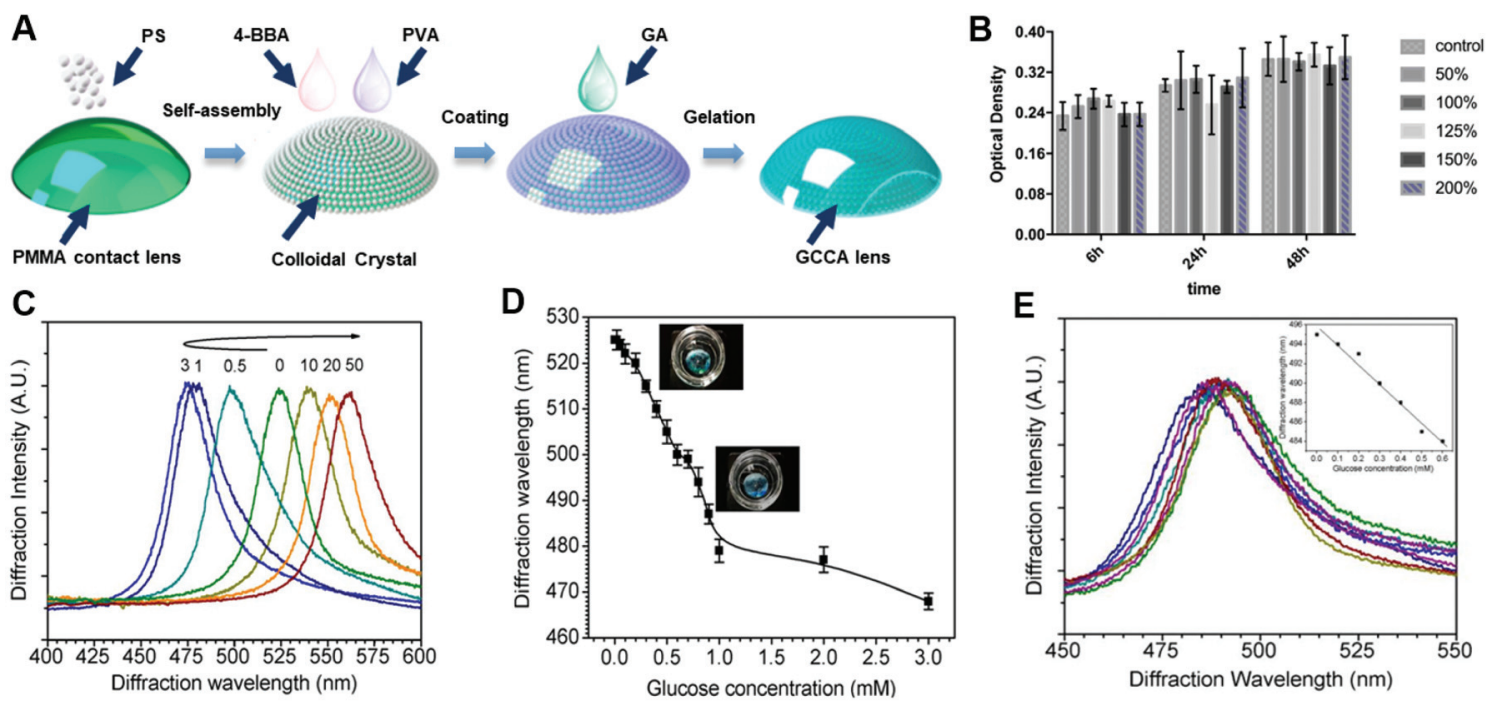

Fig. 17 The overall results and fabrication process of glucose-sensitive crystalline colloidal array contact lens sensor. (A) Fabrication process of a glucose-sensitive crystalline colloidal array contact lens sensor. During the fabrication, the self-assembled polystyrene (PS) particles were attached onto the rigid gas permeable (RGP) poly(methyl methacrylate) (PMMA) contact lenses. The homogeneous glucose-sensitive 4-boronobenzaldehyde-poly(vinyl alcohol) (4-BBA-PVA) solution was then coated onto the colloidal crystal array (CCA) to construct a physical gel. After that, $10 \%$ glutaraldehyde (GA) was cross-linked for gelation. The finalized glucose-detective gelated CCA-lens (GCCA) was washed with ultrapure water and was fabricated then. (B) The results of biocompatibility examination of GCCA-lens on Human corneal epithelial cells (HCECs). The cytotoxicity was obtained through CCK- 8 assay. (C) The diffraction shift results under visible colour range of GCCA-lens, within a glucose concentration range of 0-50 mM under buffered solution. (D) The diffraction response within the visible blue from 0 to $3 \mathrm{mM}$ of glucose. (E) The resulting diffraction wavelength shift under the physiological range of tear glucose $(0.1-0.6 \mathrm{mM})$, accompanied by the correlation curve between diffraction wavelength and glucose concentrations. Copyright 2017, Polymers. 
centre of the mould were cleared. A precursor solution was then formed containing 2-hydroxyethyl methacrylate, methylene-bis-acrylamide and 1\% v/v 2,2-diethoxyacetophenone. This was infiltrated into the colloidal crystal templates. Then, the inside mould was placed on the outside mould with a curved space left. Subsequently, the precursor solution was photopolymerized in a UV curing machine. Then the hydrogel composite-shaped contact lens was peeled off and immersed in a $4 \% \mathrm{v} / \mathrm{v}$ hydrofluoric acid (HF) solution, after which it was washed with deionized water, as the colloidal crystal template and the residual HF needed to be removed. Through this process, the structured coloured contact lens sensor was created.

An equation was implemented for the evaluation of the lens sensors' ability to monitor moisture, (eqn (7)):

$$
P=\frac{\left(W-W_{\mathrm{d}}\right)}{W} \times 100 \%
$$

where $P$ is the water content, $W$ is the original weight of the coloured contact lens sensor sufficiently swelled in water, $W_{\mathrm{d}}$ is the initial weight of the sample when $W$ is dried completely in an oven.

A digital camera and spectrometer were utilized to record the original colour and reflectance spectra of the red-colour contact lens sensor swelled in water, as well as of those dried in room temperature air under $89 \%$ humidity for different durations. Moreover, the air-dried measurements were also used to quantify the loss of water within the sensors. The plotted wavelengths of the reflectance peak with different water losses were used to obtain a relationship between the coloured contact lens sensors and the colour change within the sensor in various moistures. Furthermore, as the key indicator for dry eye disease is the amount of tear secretion, the research group placed the original sensor in eyeball moulds to simulate normal ocular conditions, as well as pathogenic conditions for xerophthalmia by adding $5 \mu \mathrm{L}$ and $15 \mu \mathrm{L}$ normal saline per 5 min (Fig. 18). ${ }^{136}$ As for the performance of coloured contact lens sensors for pressure monitoring, the original red colour sensor was put onto the contact lens mould, and then the loading units, each weighing $5.0 \mathrm{~g}$, were placed onto the mould in order to exert a certain amount of pressure onto the sensor. Finally, the reflectance spectrum and colour change with different gradients of exerted pressure were recorded by a spectrometer and a microscope (Ni-U, Nikon) (Fig. 18). Hence, the constructed coloured contact lens sensor offers great potential as a simple process for the diagnosis of various ocular diseases, and will be helpful for the POC platform.

\section{Evaluation and discussion}

Ophthalmology-related technologies have gained widespread attention in the last decade. Numerous novel techniques have been developed for ocular disease diagnosis, from imaging spectroscopies to different types of contact lens sensors. Based on the cameras discussed for external ocular photography, the possibility of integration with cameras on smartphones has been proposed. With the application of smartphone imaging, spectroscopy technologies can be applied on handheld devices, which will be helpful for real-time monitoring on point-of-care platforms. Moreover, a time-dependent dynamic monitor could be achieved by SLO, with potential applications in the diagnosis of specific ophthalmic diseases. Novel imaging techniques using auto-fluorescence can be applied to a larger research space within tears. Furthermore, contact lens tear sensing has been well explored and established in laboratory-based studies, some of which have obtained positive results in vitro and in vivo environments. However, the types of analytes detected within tears are still limited, and the detection of glucose and ions in tear fluid is the most popular and well-established technique. ${ }^{98,101,126,132}$ Proteins and enzymes could be studied further for tear analysis and subsequent diagnosis. Furthermore, different means of tear fluid sensing, such as microfluidic systems, paper-based detection, three-dimensional (3D) printing, and lateral flow assays, can also be studied and combined for improved analysis and personalized treatments. ${ }^{143}$ Hence as an intermediate direction within ophthalmologic field, some more complex biomarkers could be explored and developed with different means of detection methods for tear sensing, especially for some neuro-related diseases such as BDNF for Alzheimer's disease. On the other hand, multi-sensing of various biomarkers for one specific ocular-related disease could also be explored for obtaining accurate and reliable data for patient at point-of-care platform. However, prior to designing new contact lens sensors, it is important to consider existing manufacturing techniques, consumer demand and economic feasibility.

Initially, large volumes of contact lenses were manufactured from blown glass, which was grinded and polished to obtain the desired shape. With developments in contact lens manufacturing, there are now three techniques adopted by the majority of companies: lathing, spin coating and cast moulding. Lathing employs a dehydrated polymerized soft contact lens material with a cylindrical disk. Contact lens are produced in a designed configuration after cutting and polishing. In the spin casting manufacture process, a single concave mould is utilized to form a surface of the front lens. It involves an automatic three-step process which includes mould wetting for hydration; lens spinning and curing for packaging; and lens hydration and release for finalized manufacturing. In cast moulding, both concave and convex mould pieces are applied to fabricate the front and back surfaces of contact lenses. Due to the tremendous number of contact lens users, their potential application in minimally invasive ocular prosthetic devices has received considerable attention globally. The contact lens market worldwide is predicted to increase to over 19 billion US dollars by $2024 .{ }^{44}$ Countries including the UK, US, China, Singapore, Malaysia and Korea, have started to fabricate contact lenses not only for existing therapeutic and cosmetic uses, but also as potential medical devices with applications in a wider range of disease diagnoses and drug deliveries. 
A $\quad 0 \%$

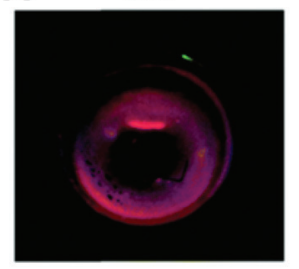

B
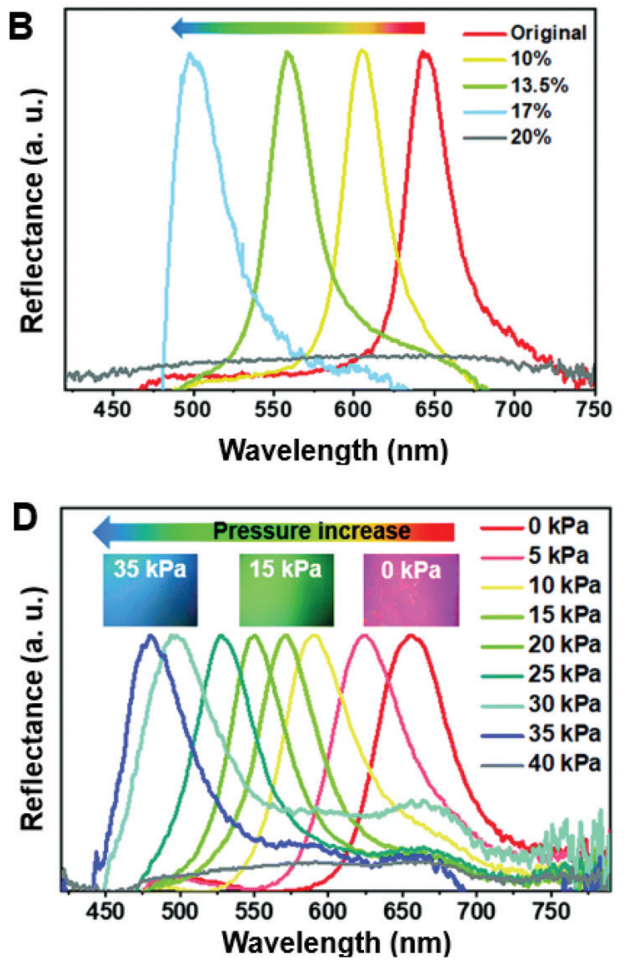

$10 \%$
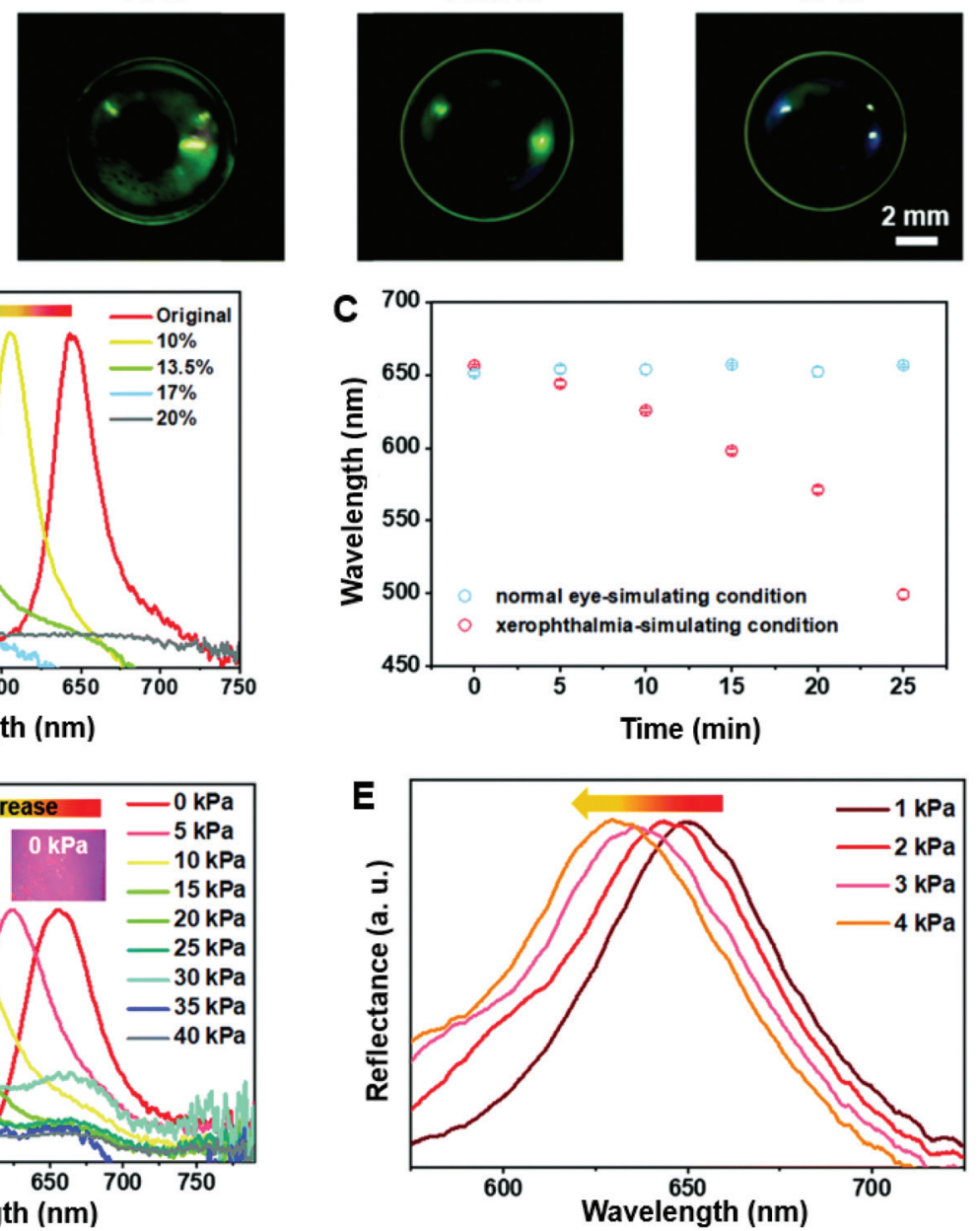

Fig. 18 Coloured contact lens sensors with the variation in moisture contents and pressures. (A) Photographs illustrating the original structurally red coloured contact lens sensor sufficiently swollen in water and its change in colour with different water loss percentages. (B) The reflectance spectra of the original red coloured contact lens sensor with different water loss percentages. (C) Plot showing the changes in the wavelengths of the reflectance peaks for the structurally coloured contact lens sensors over time under different conditions (normal eye-simulating or xerophthalmia-simulating conditions). (D) The reflectance spectra and colours of the original red coloured contact lens sensor under different levels of pressure. (E) The reflectance spectra of the red coloured contact lens sensor under pressures within the range of 1-4 kPa. Copyright 2020, Royal Society of Chemistry.

Moreover, the development of minimally invasive contact lens sensing devices facilitates the use of novel types of handheld readout electronic devices. This newly generated market of smart contact lens sensors had a value of 59.9 million USD in 2018, and is expected to exceed 1 billion USD by $2022 .^{44}$ Overall, contact lens sensors form a promising potential market for both manufacturers and patients globally.

Although tear fluid has been considered as potential media for different types of biomarkers sensing, the disadvantageous of tears sensing on the other hand is still not negligible. For example, some tears glucose sensing were considered to be less reliable compare to blood glucose detection due to the low volume and concentration sampling of glucose. ${ }^{144,145}$ Moreover, the evaporation of tear sample and in vitro detection during the analysis of tear biomarkers should be considered, especially for some low concentration contained within tears such as proteins and enzymes. Hence, more functions of in situ contact lens sensing in tear fluid can be explored as diagnostic media for indicating and continuously monitoring ocular diseases in POC settings. One important prospective research area is drug dosage and delivery within contact lens sensors, and this is expected to be achieved ultimately. Microneedle arrays have been well-established in transdermal drug delivery, ${ }^{146,147}$ chemical biosensing and as neural interfaces. ${ }^{148}$ The fabrication processes of microneedles are easy to operate using relatively low-cost 3D printing technology, and transdermal drug delivery can be achieved on minimal invasive platforms. ${ }^{149-152}$ Therefore, a future prospect for creating microneedle arrays using 3D printing technologies on contact lens sensors may be explored. Moreover, the certain ocular disease therapy might require the direct targeting into some intra-ocular positions, and some of the neuro-related diseases 
such as glaucoma would require the treatment from aqueous humor as it was stated. By achieving this, the smart contact lens can be applied not only in the tear fluid sensing stage, but also for intra-ocular treatments for ocular related diseases on POC platforms.

\section{Conclusion}

Ocular disease diagnosis is an emerging research topic worldwide. Existing developed diagnostic spectroscopies include retinoscopy, external eye photography, gonioscopy and some novel imaging technologies, such as cSLO and OCT. With these applications, multi-dimensional images can be achieved to monitor physiological or pathological conditions in precise parts of the eye, which can provide ophthalmologists and researchers with more informative data. For example, the utilization of the iPhone $4 / 4$ s with spectroscopy to photograph the eye created the potential to use smartphone readout devices in ocular diagnostics. The imaging technique employed by OCT can facilitate the differentiation of the types and severity of AMD and glaucoma. However, there is a wider potential within tear fluid sensing to be explored, with wide ranging diagnostic applications, including in cancers, neurological disorders, multiple sclerosis, systemic sclerosis and Parkinson's disease.

Various indices have been studied including glucose, IOP, $\mathrm{pH}$, corneal surface temperature and water contents, and different electrolytes within tears. Both in vivo and in vitro research has been carried out, based on the different sensing principles of fluorescent, colorimetric, electrochemical, and photonic crystal-based sensors. Amongst these sensing technologies, colorimetric sensing can exhibit a wider potential detection range. Some proteins in tear fluid are potential biomarkers for the detection and monitoring of disease, for example BDNF for glaucoma monitoring. Incorporating biosensing with biological assays could be explored in the future for ocular disease diagnosis.

In addition, to ensure patient compliance, contact lens sensors must be portable and have sufficient sensitivity, selectivity, accuracy, and reproductivity. Therefore, novel materials and sensing principles need to be explored. Moreover, smart readout devices, such as smartphones, digital cameras and laptops, need to be developed to produce informative readable data for patients on POC platforms.

In conclusion, ophthalmological diagnosis via minimally invasive tear sensing platforms is important for future research. Contact lens sensors are an emerging research direction in this area, and there is a need to further investigate possible materials, sensing techniques, and smart readout devices for data analysis in order to fulfil their functions. Future clinical applications of contact lens sensors in diagnosis, monitoring and management may help to ease pressures on healthcare systems and staff, as well as improve patient experiences. A future goal of drug dosage and delivery within contact lens sensors is expected.

\section{Author contributions}

Yuqi Shi: Organizing and writing-original manuscript, reviewing and editing. Nan Jiang: Writing-reviewing and editing, supervision. Priyanka Bikkannavar: Reviewing and editing. M. Francesca Cordeiro: Organizing and reviewing. Ali K. Yetisen: Supervision.

\section{Conflicts of interest}

The authors declare no conflicts of interest.

\section{Acknowledgements}

A. K. Y. thanks the Engineering and Physical Sciences Research Council (EPSRC) for a New Investigator Award (EP/T013567/1). N. J. acknowledges the Fundamental Research Funds for the Central Universities of China (YJ202152). Y. S. acknowledges BioRender.com for offering sources of ocular structures.

\section{References}

1 J. Enoch, L. McDonald, L. Jones, P. R. Jones and D. P. Crabb, Evaluating Whether Sight Is the Most Valued Sense, JAMA Ophthalmol., 2019, 137(11), 1317-1320.

2 J. N. Tinsley, M. I. Molodtsov, R. Prevedel, D. Wartmann, J. Espigulé-Pons, M. Lauwers and A. Vaziri, Direct detection of a single photon by humans, Nat. Commun., 2016, 7(1), 12172.

3 T. R. Fricke, N. Tahhan, S. Resnikoff, E. Papas, A. Burnett, S. M. Ho, T. Naduvilath and K. S. Naidoo, Global Prevalence of Presbyopia and Vision Impairment from Uncorrected Presbyopia: Systematic Review, Meta-analysis, and Modelling, Ophthalmology, 2018, 125(10), 1492-1499.

4 R. R. A. Bourne, S. R. Flaxman, T. Braithwaite, M. V. Cicinelli, A. Das, J. B. Jonas, J. Keeffe, J. H. Kempen, J. Leasher, H. Limburg, K. Naidoo, K. Pesudovs, S. Resnikoff, A. Silvester, G. A. Stevens, N. Tahhan, T. Y. Wong, H. R. Taylor, R. Bourne, P. Ackland, A. Arditi, Y. Barkana, B. Bozkurt, T. Braithwaite, A. Bron, D. Budenz, F. Cai, R. Casson, U. Chakravarthy, J. Choi, M. V. Cicinelli, N. Congdon, R. Dana, R. Dandona, L. Dandona, A. Das, I. Dekaris, M. Del Monte, J. Deva, L. Dreer, L. Ellwein, M. Frazier, K. Frick, D. Friedman, J. Furtado, H. Gao, G. Gazzard, R. George, S. Gichuhi, V. Gonzalez, B. Hammond, M. E. Hartnett, M. He, J. Hejtmancik, F. Hirai, J. Huang, A. Ingram, J. Javitt, J. Jonas, C. Joslin, J. Keeffe, J. Kempen, M. Khairallah, R. Khanna, J. Kim, G. Lambrou, V. C. Lansingh, P. Lanzetta, J. Leasher, J. Lim, H. Limburg, K. Mansouri, A. Mathew, A. Morse, B. Munoz, D. Musch, K. Naidoo, V. Nangia, M. Palaiou, M. B. Parodi, F. Y. Pena, K. Pesudovs, T. Peto, H. Quigley, M. Raju, P. Ramulu, S. Resnikoff, A. Robin, L. Rossetti, J. Saaddine, M. Y. A. Sandar, J. Serle, T. Shen, R. Shetty, 
P. Sieving, J. C. Silva, A. Silvester, R. S. Sitorus, D. Stambolian, G. Stevens, H. Taylor, J. Tejedor, J. Tielsch, M. Tsilimbaris, J. van Meurs, R. Varma, G. Virgili, J. Volmink, Y. X. Wang, N.-L. Wang, S. West, P. Wiedemann, T. Wong, R. Wormald and Y. Zheng, Magnitude, temporal trends, and projections of the global prevalence of blindness and distance and near vision impairment: a systematic review and meta-analysis, Lancet Glob. Health, 2017, 5(9), e888-e897.

5 A. Gordois, H. Cutler, L. Pezzullo, K. Gordon, A. Cruess, S. Winyard, W. Hamilton and K. Chua, An estimation of the worldwide economic and health burden of visual impairment, Glob. Public Health, 2012, 7(5), 465-481.

6 J. Köberlein, K. Beifus, C. Schaffert and R. P. Finger, The economic burden of visual impairment and blindness: a systematic review, BMJ Open, 2013, 3(11), e003471e003471.

7 P. B. Luppa, C. Müller, A. Schlichtiger and H. Schlebusch, Point-of-care testing (POCT): Current techniques and future perspectives, TrAC, Trends Anal. Chem., 2011, 30(6), 887-898.

8 A. J. Bandodkar and J. Wang, Non-invasive wearable electrochemical sensors: a review, Trends Biotechnol., 2014, 32(7), 363-371.

9 M. D. Steinberg, P. Kassal and I. M. Steinberg, System Architectures in Wearable Electrochemical Sensors, Electroanalysis, 2016, 28(6), 1149-1169.

10 X. Wang, Z. Liu and T. Zhang, Flexible Sensing Electronics for Wearable/Attachable Health Monitoring, Small, 2017, 13(25), 1602790.

11 S. Hagan, E. Martin and A. Enríquez-de-Salamanca, Tear fluid biomarkers in ocular and systemic disease: potential use for predictive, preventive and personalised medicine, EPMA J., 2016, 7(1), 15.

12 I. Cordero, Understanding and looking after a retinoscope and trial lens set, Community Eye Health, 2017, 30(98), 40.

13 T. J. Bennett and C. J. Barry, Ophthalmic imaging today: an ophthalmic photographer's viewpoint - a review, Clin. Exp. Ophthalmol., 2009, 37(1), 2-13.

14 D. P. Edward, in Glaucoma, ed. T. S. Vajaranant, Oxford University Press, New York, 2013.

15 R. N. Weinreb, T. Aung and F. A. Medeiros, The Pathophysiology and Treatment of Glaucoma: A Review, J. Am. Med. Assoc., 2014, 311(18), 1901-1911.

16 L. Y. Ye, L. H. Jiang, L. H. Zhang, L. C. Karp, L. J. Zhong, L. A. Tao, L. Y. Shao and L. J. Wang, Resolution of SlitLamp Microscopy Photography Using Various Cameras, Eye Contact Lens, 2013, 39(3), 205-213.

17 J. F. Salmon, 15 - Gonioscopy, in Glaucoma (Second Edition), ed. T. M. Shaarawy, M. B. Sherwood, R. A. Hitchings and J. G. Crowston, W.B. Saunders, 2015, pp. 169-178.

18 S. Park, K. Sung, S. Kang, J. Jo, K. Lee and M. Kook, Assessment of narrow angles by gonioscopy, Van Herick method and anterior segment optical coherence tomography, Jpn. J. Ophthalmol., 2011, 55(4), 343-350.
19 L. M. Sakata, R. Lavanya, D. S. Friedman, H. T. Aung, H. Gao, R. S. Kumar, P. J. Foster and T. Aung, Comparison of Gonioscopy and Anterior Segment Ocular Coherence Tomography in Detecting Angle Closure in Different Quadrants of the Anterior Chamber Angle, Ophthalmology, 2008, 115(5), 769-774.

20 C. Schönfeldt-Lecuona, T. Kregel, A. Schmidt, E. H. Pinkhardt, F. Lauda, J. Kassubek, B. J. Connemann, R. W. Freudenmann and M. Gahr, From Imaging the Brain to Imaging the Retina: Optical Coherence Tomography (OCT) in Schizophrenia, Schizophr. Bull., 2016, 42(1), 9-14.

21 M. F. Shirazi, R. E. Wijesinghe, N. K. Ravichandran, P. Kim, M. Jeon and J. Kim, Dual-path handheld system for cornea and retina imaging using optical coherence tomography, Opt. Rev., 2017, 24(2), 219-225.

22 S. Marschall, B. Sander, M. Mogensen, T. M. Jørgensen and P. E. Andersen, Optical coherence tomographycurrent technology and applications in clinical and biomedical research, Anal. Bioanal. Chem., 2011, 400(9), 2699-2720.

23 V. Mazlin, P. Xiao, E. n. Dalimier, K. Grieve, K. Irsch, J.-A. Sahel, M. Fink and A. C. Boccara, In vivo high resolution human corneal imaging using full-field optical coherence tomography, Biomed. Opt. Express, 2018, 9(2), 557568.

24 X. e. Chen, F. e. Shi and H. e. Chen, SpringerLink, Retinal Optical Coherence Tomography Image Analysis, 1st 2019 edn, 2019.

25 A. Tao, Y. Shao, J. Zhong, H. Jiang, M. Shen and J. Wang, Versatile optical coherence tomography for imaging the human eye, Biomed. Opt. Express, 2013, 4(7), 1031-1044.

26 R. Bernardes, in Optical coherence tomography: a clinical and technical update, ed. J. Cunha-Vaz, Springer, Dordrecht, 2012.

27 A. F. Low, G. J. Tearney, B. E. Bouma and I.-K. Jang, Technology Insight: optical coherence tomographycurrent status and future development, Nat. Clin. Pract. Cardiovasc. Med., 2006, 3(3), 154-162.

28 Q. X. Li, Q. Q. Fu, S. W. Shi, Y. F. Wang, J. J. Xie, X. Yu, $\mathrm{X}$. Cheng and Y. H. Liao, Relationship between plasma inflammatory markers and plaque fibrous cap thickness determined by intravascular optical coherence tomography, Heart, 2010, 96(3), 196.

29 M. J. McCabe and J. K. Croce, Optical Coherence Tomography, Circulation, 2012, 126(17), 2140-2143.

30 M. Mogensen, L. Thrane, T. M. Jørgensen, P. E. Andersen and G. B. E. Jemec, OCT imaging of skin cancer and other dermatological diseases, J. Biophotonics, 2009, 2(6-7), 442-451.

31 R. A. McLaughlin, L. Scolaro, P. Robbins, C. Saunders, S. L. Jacques and D. D. Sampson, Parametric imaging of cancer with optical coherence tomography, J. Biomed. opt., 2010, 15(4), 046029-046029.

32 C. G. Pieroni, A. J. Witkin, T. H. Ko, J. G. Fujimoto, A. Chan, J. S. Schuman, H. Ishikawa, E. Reichel and 
J. S. Duker, Ultrahigh resolution optical coherence tomography in non-exudative age related macular degeneration, Br. J. Ophthalmol., 2006, 90(2), 191-197.

33 S. Chandra, R. Rasheed, P. Sen, D. Menon and S. Sivaprasad, Inter-rater reliability for diagnosis of geographic atrophy using spectral domain OCT in age-related macular degeneration, Eye, 2021, DOI: 10.1038/s41433021-01490-5.

34 H. Sudkamp, C. von der Burchard, M. Moltmann, P. Koch, M. vom Endt, M. Münst, C. Ehlken, T. Kepp, D. TheisenKunde, R. Birngruber, J. Roider and G. Hüttmann, Selfexamination low-cost full-field OCT (SELFF-OCT) allows treatment decision for age related macular degeneration (AMD) and diabetic macular edema (DME) with high sensitivity and specificity: clinical pilot study, SPIE, 2021, vol. 11623.

35 J. P. Ehlers, J. Clark, A. Uchida, N. Figueiredo, A. Babiuch, K. E. Talcott, L. Lunasco, T. K. Le, X. Meng, M. Hu, J. Reese and S. K. Srivastava, Longitudinal Higher-Order OCT Assessment of Quantitative Fluid Dynamics and the Total Retinal Fluid Index in Neovascular AMD, Transl. Vis. Sci. Technol., 2021, 10(3), 29-29.

36 C. R. Baumal, Imaging in Diabetic Retinopathy, in Current Management of Diabetic Retinopathy, ed. C. R. Baumal and J. S. Duker, Elsevier, 2018, pp. 25-36.

37 N. T. Choplin, E. R. Craven, N. J. Reus, H. G. Lemij and H. Barnebey, 21 - Retinal Nerve Fiber Layer (RNFL) Photography and Computer Analysis, in Glaucoma (Second Edition), ed. T. M. Shaarawy, M. B. Sherwood, R. A. Hitchings and J. G. Crowston, W.B. Saunders, 2015, pp. 244-260.

38 A. Roorda, F. Romero-Borja, W. J. Donnelly III, H. Queener, T. J. Hebert and M. C. W. Campbell, Adaptive optics scanning laser ophthalmoscopy, Opt. Express, 2002, 10(9), 405-412.

39 A. Z. Nghiem, P. Nderitu, M. Lukic, M. Khatun, R. Largan, K. Kortuem, K. Balaskas and D. Sim, Comparing diabetic retinopathy lesions in scanning laser ophthalmoscopy and colour fundus photography, Acta Ophthalmol., 2019, 97(8), e1035-e1040.

40 B. Zhang, N. Li, J. Kang, Y. He and X.-M. Chen, Adaptive optics scanning laser ophthalmoscopy in fundus imaging, a review and update, Int. J. Ophthalmol., 2017, 10(11), 1751-1758.

41 Y. J. Sepah, A. Akhtar, M. A. Sadiq, Y. Hafeez, H. Nasir, B. Perez, N. Mawji, D. J. Dean, D. Ferraz and Q. D. Nguyen, Fundus autofluorescence imaging: Fundamentals and clinical relevance, Saudi J. Ophthalmol., 2014, 28(2), 111-116.

42 M. Yung, M. A. Klufas and D. Sarraf, Clinical applications of fundus autofluorescence in retinal disease, Int. J. Retin. Vitr., 2016, 2(1), 12.

43 E. J. Warrant and D.-E. Nilsson, Absorption of white light in photoreceptors, Vision Res., 1998, 38(2), 195-207.

44 R. Moreddu, D. Vigolo and A. K. Yetisen, Contact Lens Technology: From Fundamentals to Applications, Adv. Healthcare Mater., 2019, 8(15), 1900368.
45 VisionDirect Types of Contact Lenses, https://www. visiondirect.co.uk/eye-care-centre/types-of-contact-lenses (accessed 3/2).

46 N. M. Farandos, A. K. Yetisen, M. J. Monteiro, C. R. Lowe and S. H. Yun, Contact Lens Sensors in Ocular Diagnostics, Adv. Healthcare Mater., 2015, 4(6), 792-810.

47 T. H. Cedarstaff and A. Tomlinson, A comparative study of tear evaporation rates and water content of soft contact lenses, Am. J. Optom. Physiol. Opt., 1983, 60(3), 167-174.

48 National Research Council Working Group on Contact Lens Use Under Adverse Conditions, in Considerations in Contact Lens Use Under Adverse Conditions: Proceedings of a Symposium, ed. P. Ebert Flattau, National Academies Press, US. Copyright (C) National Academy of Sciences, Washington, DC, 1991.

49 R. Moreddu, M. Elsherif, H. Butt, D. Vigolo and A. K. Yetisen, Contact lenses for continuous corneal temperature monitoring, RSC Adv., 2019, 9(20), 11433-11442.

50 R. S. Riaz, M. Elsherif, R. Moreddu, I. Rashid, M. U. Hassan, A. K. Yetisen and H. Butt, AnthocyaninFunctionalized Contact Lens Sensors for Ocular pH Monitoring, ACS Omega, 2019, 4(26), 21792-21798.

51 A. K. Yetisen, N. Jiang, G. C. M. Castaneda, Z. I. Erenoglu, J. Dong, X. Dong, S. Stößer, M. Brischwein, H. Butt, M. F. Cordeiro, M. Jakobi, O. Hayden and A. W. Koch, Scleral lens sensor for ocular electrolyte analysis, $A d v$. Mater., 2019, 1906762-1906762.

52 I. E. Araci, B. Su, S. R. Quake and Y. Mandel, An implantable microfluidic device for self-monitoring of intraocular pressure, Nat. Med., 2014, 20(9), 1074-1078.

53 M. Moshirfar, K. Pierson, K. Hanamaikai, L. SantiagoCaban, V. Muthappan and S. F. Passi, Artificial tears potpourri: a literature review, Clin. Ophthalmol., 2014, 8, 1419-1433.

$54 \mathrm{H}$. Uusitalo, Tear fluid biomarkers, conjunctival inflammation in glaucoma, Acta Ophthalmol., 2016, 7(1), 15-15.

55 Otsuka Functions of Tears and How they work, https:// www.otsuka.co.jp/en/health-and-illness/dry-eye/functionsof-tears/(accessed 3/2).

56 M. Elsherif, A. E. Salih, A. K. Yetisen and H. Butt, Contact Lenses for Color Vision Deficiency, Adv. Mater. Technol., 2021, 6(1), 2000797.

57 A. E. Salih, M. Elsherif, F. Alam, A. K. Yetisen and H. Butt, Gold Nanocomposite Contact Lenses for Color Blindness Management, ACS Nano, 2021, 15(3), 4870-4880.

58 S. Dan-Na, D. Tong and Q. Wei-Qiang, Development and clinical applications of therapeutic contact lenses, IJO PRESS, 2018, 18(2), 271-274.

59 S. J. H. Lea, M. A. Z. Neugebauer, R. G. Smith and S. A. Vernon, The incidence of ophthalmic problems in the contact lens wearing population, Eye, 1990, 4(5), 706711.

60 A. K. Dumbleton, W. C. Woods, W. L. Jones and W. D. Fonn, The Impact of Contemporary Contact Lenses on Contact Lens Discontinuation, Eye Contact Lens, 2013, 39(1), 93-99. 
61 A. Solomon, Allergic manifestations of contact lens wearing, Curr. Opin. Allergy Clin. Immunol., 2016, 16(5), 492-497.

62 D. Harvey, N. W. Hayes and B. Tighe, Fibre optics sensors in tear electrolyte analysis: Towards a novel point of care potassium sensor, Contact Lens Anterior Eye, 2012, 35(3), 137-144.

63 L. Tong, L. Zhou, R. W. Beuerman, S. Z. Zhao and X. R. Li, Association of tear proteins with Meibomian gland disease and dry eye symptoms, Br. J. Ophthalmol., 2011, 95(6), 848.

64 K.-S. Na, J.-W. Mok, J. Y. Kim, C. R. Rho and C.-K. Joo, Correlations between Tear Cytokines, Chemokines, and Soluble Receptors and Clinical Severity of Dry Eye Disease, Invest. Ophthalmol. Visual Sci., 2012, 53(9), 54435450.

65 K. R. VanDerMeid, S. P. Su, K. W. Ward and J.-Z. Zhang, Correlation of Tear Inflammatory Cytokines and Matrix Metalloproteinases with Four Dry Eye Diagnostic Tests, Invest. Ophthalmol. Visual Sci., 2012, 53(3), 1512-1518.

66 A. López-Miguel, M. Tesón, V. Martín-Montañez, A. Enríquez-de-Salamanca, M. E. Stern, M. Calonge and M. J. González-García, Dry Eye Exacerbation in Patients Exposed to Desiccating Stress under Controlled Environmental Conditions, Am. J. Ophthalmol., 2014, 157(4), 788-798.e2.

67 A. López-Miguel, M. Tesón, V. Martín-Montañez, A. Enríquez-de-Salamanca, M. E. Stern, M. J. GonzálezGarcía and M. Calonge, Clinical and Molecular Inflammatory Response in Sjögren Syndrome-Associated Dry Eye Patients Under Desiccating Stress, Am. J. Ophthalmol., 2016, 161, 133-141.e2.

68 X. Tan, S. Sun, Y. Liu, T. Zhu, K. Wang, T. Ren, Z. Wu, H. Xu and L. Zhu, Analysis of Th17-associated cytokines in tears of patients with dry eye syndrome, Eye, 2014, 28(5), 608-613.

69 M. J. Benito, M. J. González-García, M. Tesón, N. García, I. Fernández, M. Calonge and A. Enríquez-de-Salamanca, Intra- and inter-day variation of cytokines and chemokines in tears of healthy subjects, Exp. Eye Res., 2014, 120, 43-49.

70 J. C. F. Pong, C. Y. Chu, K. O. Chu, T. C. W. Poon, S. M. Ngai, C. P. Pang and C. C. Wang, Identification of hemopexin in tear film, Anal. Biochem., 2010, 404(1), 8285.

71 J. C. F. Pong, C. Y. Chu, W. Y. Li, L. Y. Tang, L. Li, W. T. Lui, T. C. W. Poon, S. K. Rao, D. S. C. Lam, C. C. Wang and C. P. Pang, Association of Hemopexin in Tear Film and Conjunctival Macrophages With Vernal Keratoconjunctivitis, Arch. Ophthalmol., 2011, 129(4), 453461.

72 A. Leonardi, A. Palmigiano, E. A. Mazzola, A. Messina, E. M. S. Milazzo, M. Bortolotti and D. Garozzo, Identification of human tear fluid biomarkers in vernal keratoconjunctivitis using iTRAQ quantitative proteomics, Allergy, 2014, 69(2), 254-260.
73 M. Sacchetti, A. Micera, A. Lambiase, S. Speranza, F. Mantelli, G. Petrachi, S. Bonini and S. Bonini, Tear levels of neuropeptides increase after specific allergen challenge in allergic conjunctivitis, Mol. Vision, 2011, 17, $47-52$.

74 A. Leonardi, F. Borghesan, D. Faggian and M. Plebani, Microarray-based IgE detection in tears of patients with vernal keratoconjunctivitis, Pediatr. Allergy Immunol., 2015, 26(7), 641-645.

75 R. Shetty, A. Ghosh, R. R. Lim, M. Subramani, K. Mihir, A. R. Reshma, A. Ranganath, S. Nagaraj, R. M. Nuijts, R. Beuerman, R. Shetty, D. Das, S. S. Chaurasia, A. SinhaRoy and A. Ghosh, Elevated expression of matrix metalloproteinase-9 and inflammatory cytokines in keratoconus patients is inhibited by cyclosporine A, Invest. Ophthalmol. Visual Sci., 2015, 56(2), 738-750.

76 R. Sorkhabi, A. Ghorbanihaghjo, N. Taheri and M. H. Ahoor, Tear film inflammatory mediators in patients with keratoconus, Int. Ophthalmol., 2015, 35(4), 467-472.

77 J. You, C. Hodge, L. Wen, J. W. McAvoy, M. C. Madigan and G. Sutton, Tear levels of SFRP1 are significantly reduced in keratoconus patients, Mol. Vis., 2013, 19, 509515.

78 T. Göncü, A. Akal, F. M. Adbelli, S. Çakmak, H. Sezen and Ö.F Ylmaz, Tear Film and Serum Prolidase Activity and Oxidative Stress in Patients With Keratoconus, Cornea, 2015, 34(9), 1019-1023.

79 T. Skwor, R. P. Kandel, S. Basravi, A. Khan, B. Sharma and D. Dean, Characterization of Humoral Immune Responses to Chlamydial HSP60, CPAF, and CT795 in Inflammatory and Severe Trachoma, Invest. Ophthalmol. Visual Sci., 2010, 51(10), 5128-5136.

80 D. Gupta, J. Wen, J. Huebner, S. Stinnett, V. Kraus, H. C. Tseng and M. Walsh, Cytokine biomarkers in tear film for primary open-angle glaucoma, Clin. Ophthalmol., 2017, 11, 411-416.

81 C. Rossi, I. Cicalini, M. C. Cufaro, L. Agnifili, L. Mastropasqua, P. Lanuti, M. Marchisio, V. De Laurenzi, P. Del Boccio and D. Pieragostino, Multi-Omics Approach for Studying Tears in Treatment-Naïve Glaucoma Patients, Int. J. Mol. Sci., 2019, 20(16), 4029.

82 É. Csősz, E. Deák, N. Tóth, C. E. Traverso, A. Csutak and J. Tőzsér, Comparative analysis of cytokine profiles of glaucomatous tears and aqueous humour reveals potential biomarkers for trabeculectomy complications, FEBS Open Bio, 2019, 9(5), 1020-1028.

83 A. Naik, S. Shrivastava, N. Abidi, R. Yadav, P. Shah and Y. Gala, Study of tear proteins for possible biomarker in primary open-angle glaucoma, JCOR, 2018, 6(2), 66-70.

84 A. A. Shpak, A. B. Guekht, T. A. Druzhkova, K. I. Kozlova and N. V. Gulyaeva, Brain-Derived Neurotrophic Factor in Patients with Primary Open-Angle Glaucoma and Agerelated Cataract, Curr. Eye Res., 2018, 43(2), 224-231.

85 R. Ihnatko, U. Edén, N. Lagali, A. Dellby and P. Fagerholm, Analysis of protein composition and 
protein expression in the tear fluid of patients with congenital aniridia, J. Proteomics, 2013, 94, 78-88.

86 A. Peral, G. Carracedo and J. Pintor, Diadenosine polyphosphates in the tears of aniridia patients, Acta Ophthalmol., 2015, 93(5), e337-e342.

87 I. Cicalini, C. Rossi, D. Pieragostino, L. Agnifili, L. Mastropasqua, M. Di Ioia, G. De Luca, M. Onofrj, L. Federici and P. Del Boccio, Integrated Lipidomics and Metabolomics Analysis of Tears in Multiple Sclerosis: An Insight into Diagnostic Potential of Lacrimal Fluid, Int. J. Mol. Sci., 2019, 20(6), 1265.

88 D. Böhm, K. Keller, J. Pieter, N. Boehm, D. Wolters, W. Siggelkow, A. Lebrecht, M. Schmidt, H. Kölbl, N. Pfeiffer and F.-H. Grus, Comparison of tear protein levels in breast cancer patients and healthy controls using a de novo proteomic approach, Oncol. Rep., 2012, 28(2), 429.

89 É. Csősz, P. Boross, A. Csutak, A. Berta, F. Tóth, S. Póliska, Z. Török and J. Tőzsér, Quantitative analysis of proteins in the tear fluid of patients with diabetic retinopathy, J. Proteomics, 2012, 75(7), 2196-2204.

90 H.-J. Kim, P.-K. Kim, H.-S. Yoo and C.-W. Kim, Comparison of tear proteins between healthy and early diabetic retinopathy patients, Clin. Biochem., 2012, 45(1), 60-67.

91 C. Costagliola, V. Romano, M. De Tollis, F. Aceto, R. dell'Omo, M. R. Romano, C. Pedicino and F. Semeraro, TNF-Alpha Levels in Tears: A Novel Biomarker to Assess the Degree of Diabetic Retinopathy, Mediators Inflammation, 2013, 2013, 629529.

92 A. Hanyuda, N. Sawada, K. Yuki, M. Uchino, Y. Ozawa, M. Sasaki, K. Yamagishi, H. Iso, K. Tsubota and S. Tsugane, Relationships of diabetes and hyperglycaemia with intraocular pressure in a Japanese population: the JPHC-NEXT Eye Study, Sci. Rep., 2020, 10(1), 5355-5355.

93 A. Rentka, J. Hársfalvi, A. Berta, K. Köröskényi, Z. Szekanecz, G. Szücs, P. Szodoray and Á. Kemény-Beke, Vascular Endothelial Growth Factor in Tear Samples of Patients with Systemic Sclerosis, Mediators Inflammation, 2015, 2015, 573681.

94 A. Rentka, J. Harsfalvi, G. Szucs, Z. Szekanecz, P. Szodoray, K. Koroskenyi and A. Kemeny-Beke, Membrane array and multiplex bead analysis of tear cytokines in systemic sclerosis, Immunol. Res., 2016, 64(2), 619-626.

95 S. S. Çomoğlu, H. Güven, M. Acar, G. Öztürk and B. Koçer, Tear levels of tumor necrosis factor-alpha in patients with Parkinson's disease, Neurosci. Lett., 2013, 553, 63-67.

96 C. Salvisberg, N. Tajouri, A. Hainard, P. R. Burkhard, P. H. Lalive and N. Turck, Exploring the human tear fluid: Discovery of new biomarkers in multiple sclerosis, Proteomics: Clin. Appl., 2014, 8(3-4), 185-194.

97 R. Moreddu, J. S. Wolffsohn, D. Vigolo and A. K. Yetisen, Laser-inscribed contact lens sensors for the detection of analytes in the tear fluid, Sens. Actuators, B, 2020, 317, 128183.
98 R. Moreddu, M. Elsherif, H. Adams, D. Moschou, M. F. Cordeiro, J. S. Wolffsohn, D. Vigolo, H. Butt, J. M. Cooper and A. K. Yetisen, Integration of paper microfluidic sensors into contact lenses for tear fluid analysis, Lab Chip, 2020, 20(21), 3970-3979.

99 R. Badugu, J. R. Lakowicz and C. D. Geddes, Noninvasive Continuous Monitoring of Physiological Glucose Using a Monosaccharide-Sensing Contact Lens, Anal. Chem., 2004, 76(3), 610-618.

100 A. K. Yetisen, N. Jiang, C. M. Castaneda, Z. I. Erenoglu, J. Dong, X. Dong, S. Stößer, M. Brischwein, H. Butt, M. F. Cordeiro, M. Jakobi, O. Hayden and A. W. Koch, Scleral Lens Sensor for Ocular Electrolyte Analysis, $A d v$. Mater., 2020, 32(6), 1906762.

101 M. Elsherif, M. U. Hassan, A. K. Yetisen and H. Butt, Wearable Contact Lens Biosensors for Continuous Glucose Monitoring Using Smartphones, ACS Nano, 2018, 12(6), 5452-5462.

102 C. Zhang, G. G. Cano and P. V. Braun, Linear and fast hydrogel glucose sensor materials enabled by volume resetting agents, Adv. Mater., 2014, 26(32), 5678-5683.

103 J.-L. Ruan, C. Chen, J.-H. Shen, X.-L. Zhao, S.-H. Qian and Z.-G. Zhu, A Gelated Colloidal Crystal Attached Lens for Noninvasive Continuous Monitoring of Tear Glucose, Polymers, 2017, 9(4), 125.

104 H. Yao, Y. Liao, A. R. Lingley, A. Afanasiev, I. Lähdesmäki, B. P. Otis and B. A. Parviz, A contact lens with integrated telecommunication circuit and sensors for wireless and continuous tear glucose monitoring, J. Micromech. Microeng., 2012, 22(7), 075007.

105 A. F. Ogata, S.-W. Song, S.-H. Cho, W.-T. Koo, J.-S. Jang, Y. J. Jeong, M.-H. Kim, J. Y. Cheong, R. M. Penner and I.-D. Kim, An Impedance-Transduced Chemiresistor with a Porous Carbon Channel for Rapid, Nonenzymatic, Glucose Sensing, Anal. Chem., 2018, 90(15), 9338-9346.

106 N. Thomas, I. Lähdesmäki and B. A. Parviz, A contact lens with an integrated lactate sensor, Sens. Actuators, B, 2012, 162(1), 128-134.

107 H. Maeda and P. Anzenbacher Jr., Colorimetric Sensors, in Supramolecular Chemistry, ed. P. A. Gale and J. W. Steed, 2012.

108 R. G. Mortimer, 20 - Spectroscopy and Photochemistry, in Physical Chemistry, ed. R. G. Mortimer, Academic Press, Burlington, 2nd edn, 2000, pp. 751-815.

109 E. F. M. Gabriel, P. T. Garcia, T. M. G. Cardoso, F. M. Lopes, F. T. Martins and W. K. T. Coltro, Highly sensitive colorimetric detection of glucose and uric acid in biological fluids using chitosan-modified paper microfluidic devices, Analyst, 2016, 141(15), 4749-4756.

110 P. de Tarso Garcia, T. M. Garcia Cardoso, C. D. Garcia, E. Carrilho and W. K. Tomazelli Coltro, A handheld stamping process to fabricate microfluidic paper-based analytical devices with chemically modified surface for clinical assays, RSC Adv. , 2014, 4(71), 37637-37644.

111 E. F. M. Gabriel, P. T. Garcia, F. M. Lopes and W. K. T. Coltro, Paper-Based Colorimetric Biosensor for 
Tear Glucose Measurements, Micromachines, 2017, 8(4), 104.

112 C. M. Cheng, A. W. Martinez, J. Gong, C. R. Mace, S. T. Phillips, E. Carrilho, K. A. Mirica and G. M. Whitesides, Paper-based ELISA, Angew. Chem., Int. Ed. Engl., 2010, 49(28), 4771-4774.

113 J. Hu, S. Wang, L. Wang, F. Li, B. Pingguan-Murphy, T. J. Lu and F. Xu, Advances in paper-based point-of-care diagnostics, Biosens. Bioelectron., 2014, 54, 585-597.

114 M.-Y. Hsu, C.-Y. Yang, W.-H. Hsu, K.-H. Lin, C.-Y. Wang, Y.-C. Shen, Y.-C. Chen, S.-F. Chau, H.-Y. Tsai and C.-M. Cheng, Monitoring the VEGF level in aqueous humor of patients with ophthalmologically relevant diseases via ultrahigh sensitive paper-based ELISA, Biomaterials, 2014, 35(12), 3729-3735.

115 X. Zhu, S. Xiong, J. Zhang, X. Zhang, X. Tong and S. Kong, Improving paper-based ELISA performance through covalent immobilization of antibodies, Sens. Actuators, B, 2018, 255, 598-604.

116 A. Ghaffariyeh, N. Honarpisheh, Y. Shakiba, S. Puyan, T. Chamacham, F. Zahedi and M. Zarrineghbal, Brainderived neurotrophic factor in patients with normaltension glaucoma, Optometry - J. Am. Optom. Assoc., 2009, 80(11), 635-638.

117 C. E. Wayne, Photochemistry, Oxford University Press, Oxford, New York, 1996.

118 R. Badugu, B. H. Jeng, E. A. Reece and J. R. Lakowicz, Contact lens to measure individual ion concentrations in tears and applications to dry eye disease, Anal. Biochem., 2018, 542, 84-94.

119 R. Badugu, H. Szmacinski, E. A. Reece, B. H. Jeng and J. R. Lakowicz, Fluorescent Contact Lens for Continuous Non-invasive Measurements of Sodium and Chloride Ion Concentrations in Tears, Anal. Biochem., 2020, 113902.

120 R. Badugu, E. A. Reece and J. Lakowicz, Glucose-sensitive silicone hydrogel contact lens toward tear glucose monitoring, J. Biomed. Opt., 2018, 23(5), 057005.

121 K. Maejima, S. Tomikawa, K. Suzuki and D. Citterio, Inkjet printing: an integrated and green chemical approach to microfluidic paper-based analytical devices, RSC Adv., 2013, 3(24), 9258-9263.

122 K. Yamada, T. G. Henares, K. Suzuki and D. Citterio, Distance-Based Tear Lactoferrin Assay on Microfluidic Paper Device Using Interfacial Interactions on SurfaceModified Cellulose, ACS Appl. Mater. Interfaces, 2015, $7(44), 24864-24875$.

123 H. Yao, A. J. Shum, M. Cowan, I. Lähdesmäki and B. A. Parviz, A contact lens with embedded sensor for monitoring tear glucose level, Biosens. Bioelectron., 2011, 26(7), 3290-3296.

124 H. Yao, Y. Liao, A. R. Lingley, A. Afanasiev, I. Lähdesmäki, B. P. Otis and B. A. Parviz, A contact lens with integrated telecommunication circuit and sensors for wireless and continuous tear glucose monitoring, J. Micromech. Microeng., 2012, 22(7), 075007.
125 M. Chu, T. Shirai, D. Takahashi, T. Arakawa, H. Kudo, K. Sano, S.-i. Sawada, K. Yano, Y. Iwasaki, K. Akiyoshi, M. Mochizuki and K. Mitsubayashi, Biomedical soft contact-lens sensor for in situ ocular biomonitoring of tear contents, Biomed. Microdevices, 2011, 13(4), 603-611.

126 M. X. Chu, K. Miyajima, D. Takahashi, T. Arakawa, K. Sano, S.-i. Sawada, H. Kudo, Y. Iwasaki, K. Akiyoshi, M. Mochizuki and K. Mitsubayashi, Soft contact lens biosensor for in situ monitoring of tear glucose as non-invasive blood sugar assessment, Talanta, 2011, 83(3), 960965.

127 J. Kim, M. Kim, M.-S. Lee, K. Kim, S. Ji, Y.-T. Kim, J. Park, K. Na, K.-H. Bae, H. Kyun Kim, F. Bien, C. Young Lee and J.-U. Park, Wearable smart sensor systems integrated on soft contact lenses for wireless ocular diagnostics, Nat. Commun., 2017, 8(1), 14997.

128 M. Ku, J. Kim, J.-e. Won, W. Kang, Y.-G. Park, J. Park, J.-H. Lee, J. Cheon, H. Lee and J.-U. Park, Smart, soft contact lens for wireless immunosensing of cortisol, Sci. $A d v .$, 2020, 6(28), eabb2891.

129 J. Kim, J. Park, Y.-G. Park, E. Cha, M. Ku, H. S. An, K.-P. Lee, M.-I. Huh, J. Kim, T.-S. Kim, D. W. Kim, H. K. Kim and J.-U. Park, A soft and transparent contact lens for the wireless quantitative monitoring of intraocular pressure, Nat. Biomed. Eng., 2021, 5(7), 772782.

130 J. Kim, J. Kim, M. Ku, E. Cha, S. Ju, W. Y. Park, K. H. Kim, D. W. Kim, P.-O. Berggren and J.-U. Park, Intraocular Pressure Monitoring Following Islet Transplantation to the Anterior Chamber of the Eye, Nano Lett., 2020, 20(3), 1517-1525.

131 J. Jang, J. Kim, H. Shin, Y. G. Park, B. J. Joo, H. Seo, J. E. Won, D. W. Kim, C. Y. Lee, H. K. Kim and J. U. Park, Smart contact lens and transparent heat patch for remote monitoring and therapy of chronic ocular surface inflammation using mobiles, Sci. Adv., 2021, 7(14), eabf7194.

132 S. Kim, H.-J. Jeon, S. Park, D. Y. Lee and E. Chung, Tear Glucose Measurement by Reflectance Spectrum of a Nanoparticle Embedded Contact Lens, Sci. Rep., 2020, 10(1), 8254.

133 M. Donora, A. Vásquez Quintero, H. De Smet and I. Underwood, Spatiotemporal electrochemical sensing in a smart contact lens, Sens. Actuators, B, 2020, 303, 127203.

134 K. Kim, H. J. Kim, H. Zhang, W. Park, D. Meyer, M. K. Kim, B. Kim, H. Park, B. Xu, P. Kollbaum, B. W. Boudouris and C. H. Lee, All-printed stretchable corneal sensor on soft contact lenses for noninvasive and painless ocular electrodiagnosis, Nat. Commun., 2021, 12(1), 1544.

135 C. Zhang, M. D. Losego and P. V. Braun, Hydrogel-Based Glucose Sensors: Effects of Phenylboronic Acid Chemical Structure on Response, Chem. Mater., 2013, 25(15), 32393250.

136 Y. Wang, Q. Zhao and X. Du, Structurally coloured contact lens sensor for point-of-care ophthalmic health monitoring, J. Mater. Chem. B, 2020, 8(16), 3519-3526. 
137 J. Ge and Y. Yin, Responsive Photonic Crystals, Angew. Chem., Int. Ed., 2011, 50(7), 1492-1522.

138 J. Teyssier, S. V. Saenko, D. van der Marel and M. C. Milinkovitch, Photonic crystals cause active colour change in chameleons, Nat. Commun., 2015, 6(1), 6368.

139 J. Hou, M. Li and Y. Song, Patterned Colloidal Photonic Crystals, Angew. Chem., Int. Ed., 2018, 57(10), 2544-2553.

140 Z. Li, M. Wang, X. Zhang, D. Wang, W. Xu and Y. Yin, Magnetic Assembly of Nanocubes for OrientationDependent Photonic Responses, Nano Lett., 2019, 19(9), 6673-6680.

141 Y. Wang, H. Cui, Q. Zhao and X. Du, Chameleon-Inspired Structural-Color Actuators, Matter, 2019, 1(3), 626-638.

142 Z. Xie, L. Li, P. Liu, F. Zheng, L. Guo, Y. Zhao, L. Jin, T. Li and Z. Gu, Self-Assembled Coffee-Ring Colloidal Crystals for Structurally Colored Contact Lenses, Small, 2015, 11(8), 926-930.

143 F. Alam, M. Elsherif, B. AlQattan, A. Salih, S. M. Lee, A. K. Yetisen, S. Park and H. Butt, 3D Printed Contact Lenses, ACS Biomater. Sci. Eng., 2021, 7(2), 794-803.

144 J. T. La Belle, A. Adams, C.-E. Lin, E. Engelschall, B. Pratt and C. B. Cook, Self-monitoring of tear glucose: the development of a tear based glucose sensor as an alternative to self-monitoring of blood glucose, Chem. Commun., 2016, 52(59), 9197-9204.

145 D. Bruen, C. Delaney, L. Florea and D. Diamond, Glucose Sensing for Diabetes Monitoring: Recent Developments, Sensors, 2017, 17(8), 1866.
146 M. A. Luzuriaga, D. R. Berry, J. C. Reagan, R. A. Smaldone and J. J. Gassensmith, Biodegradable 3D printed polymer microneedles for transdermal drug delivery, Lab Chip, 2018, 18(8), 1223-1230.

147 C. P. P. Pere, S. N. Economidou, G. Lall, C. Ziraud, J. S. Boateng, B. D. Alexander, D. A. Lamprou and D. Douroumis, 3D printed microneedles for insulin skin delivery, Int. J. Pharm., 2018, 544(2), 425-432.

148 K. J. Krieger, N. Bertollo, M. Dangol, J. T. Sheridan, M. M. Lowery and E. D. O'Cearbhaill, Simple and customizable method for fabrication of high-aspect ratio microneedle molds using low-cost 3D printing, Microsyst. Nanoeng., 2019, 5(1), 42.

149 M. Wu, Y. Zhang, H. Huang, J. Li, H. Liu, Z. Guo, L. Xue, S. Liu and Y. Lei, Assisted 3D printing of microneedle patches for minimally invasive glucose control in diabetes, Mater. Sci. Eng., C, 2020, 117, 111299.

150 C. Yeung, S. Chen, B. King, H. Lin, K. King, F. Akhtar, G. Diaz, B. Wang, J. Zhu, W. Sun, A. Khademhosseini and S. Emaminejad, A 3D-printed microfluidic-enabled hollow microneedle architecture for transdermal drug delivery, Biomicrofluidics, 2019, 13(6), 064125.

151 A. K. Yetisen, N. Jiang, A. Tamayol, G. U. Ruiz-Esparza, Y. S. Zhang, S. Medina-Pando, A. Gupta, J. S. Wolffsohn, H. Butt, A. Khademhosseini and S. -H. Yun, Paper-based microfluidic system for tear electrolyte analysis, Lab Chip, 2017, 17(6), 1137-1148.

152 N. Jiang, Y. Montelongo, H. Butt and A. K. Yetisen, Microfluidic Contact Lenses, Small, 2018, 14(15), 1704363. 